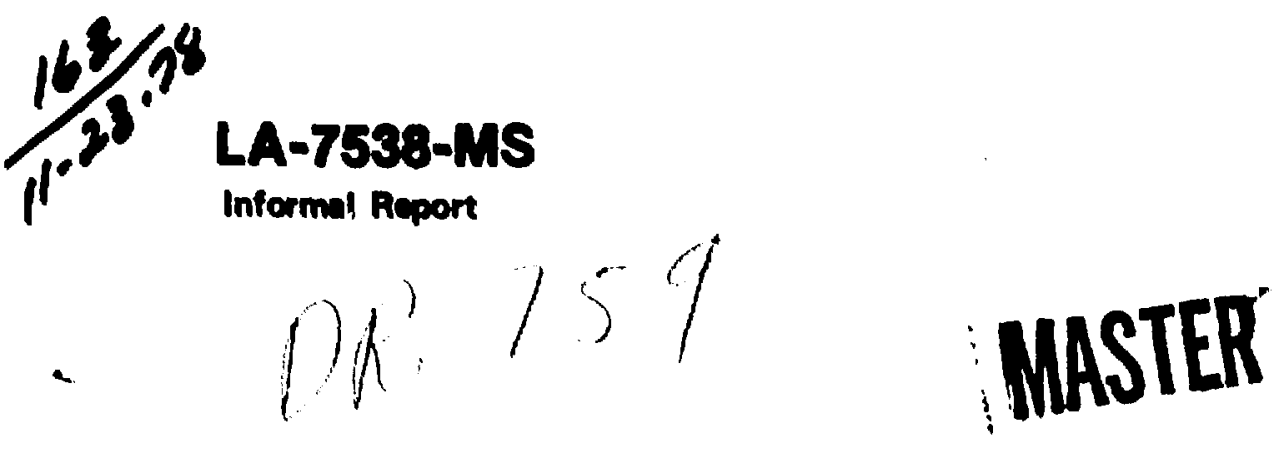

Linear and Nonlinear Symmetrically Loaded Shells of Revolution Approximated with the Finite Element Method

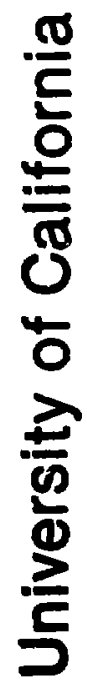


LA.7538-MS

Informal Report

UC.71

Issued: October 1978

\section{Linear and Nonlinear Symmetrically Loaded Shells of Revolution Approximated with the Finite Element Method}

William A. Cook 
PREFACE

Nuclear Material shipping containers have shells of revolution as a basic structural component. Analytically modeling the response of these containers to severe accident impact conditions requires a nonlinear shell-of-revolution model that accounts for both geometric and material nonlinearities. Present models are limited to large displacements, small rotations, and nonlinear materials.

This report discusses a first approach to developing a finite element nonlinear shell of revolution model that accounts for these nonlinear geometric effects. The approach developed accounts for large strains as well as large displacements but is limited to small rotations. It also allows the modeling of nonlinear materials.

A later report will present a nonlinear shell-ofrevolution finite alement model that will account for large displacements, large strains, large rotations, and nonlinear materials. 
CONTENTS

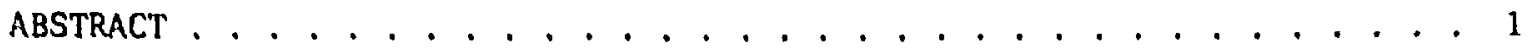

NOMENCLATURE . . . . . . . . . . . . . . . . . . . . . vi

I. INTRODUCTION AND RESULTS . . . . . . . . . . . . . . . . . . . . 1

II. LINEAR SHELLS USING POTENTIAL ENERGY VARIATIONAL PRINCIPLE. . . . . . 5

III. LINEAR SHELLS USING MIXED VARIATIONAL FPINCIPLE . . . . . . . . . . 11

IV. NONLINEAR SHELLS USING POTENTIAL ENERGY VARIATIONAL PRINCIPLE . . . 16

V. RECOMENDATIONS. . . . . . . . . . . . . . . . . . . . . . 20

APPENDIX A. B MATRIX FOR DISPLACEMENTS AND ROTATION . . . . . . . . . . 22

APPENDIX B. B MATRIX FOR DISPLACEMENTS AND MERIDIONAL MOMENT. . . . . . . 25

APPENDIX C. MIXED VARIATIONAL PRINCIPLE . . . . . . . . . . . . 27

APPENDIX D. POTENTIAL ENERGY VARIATIONAL PRINCIPLE. . . . . . . . . 30

APPENDIX E. SHAPE FUNCTIONS FOR SHELL ELEMENTS. . . . . . . . . . . 33

APPENDIX $F$. EQUILIBRIUM EQUATIONS . . . . . . . . . . . . . 37

APPENDIX G. GEOMETRIC QUANTITIES REQUIRED FOR STIFFNESS AND FORCE

MODIFICATION CALCULATIONS . . . . . . . . . . . . . . 41

APPENDIX H. GEOMETRIC NONLINEAR SHELL QUANTITIES. . . . . . . . . . 46

APPENDIX I. INCREMENTING AND LINEARIZING OF $\varepsilon_{s}, \varepsilon_{\theta}, \kappa_{s}$, AND $\kappa_{\theta}$ FOR

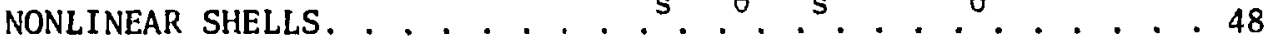

APPENDIX J. INCREMENTAL $\mathrm{N}_{\mathrm{S}}, \mathrm{N}_{\theta}, \mathrm{M}_{\mathrm{s}}$, AND $\mathrm{M}_{\theta} . \ldots . . . . . . .52$ APPENDIX K. NONLINEAR MATERIAL PROPERTIES . . . . . . . . . . 53

APPENDIX L. $\varepsilon_{\theta}$ AND $\kappa_{\theta}$ FOR CLOSED $(r=0)$ SHELL. . . . . . . . . . . 55 REFERENCES . . . . . . . . . . . . . . . . . . . 57 
NOMENCLATURE

LATIN

B - Boundary for sicll (ends)

$\stackrel{B}{=} \quad$ B matrix (see Appendix A and B) strain displacement approximation

C - Curvature $(1 / R)$

$\mathrm{C}_{\mathrm{s}}$

$\mathrm{C}_{s \theta}$

$c_{\theta}$

D

$\mathrm{D}_{\mathrm{s} \theta}$

$\mathrm{D}_{\theta} \mathrm{J}$

Shell material properties (see Chapter II)

h - Two-and three-nodal point shape functions for shells (see Appendix E)

I - Mixed variational function (see Appendix C)

L - Length of shell

$L_{e} \quad$ Length of shell finite element

$M_{S}$ - Meridional bending moment

$\bar{M}_{S}$ - Applied meridional bending moment

$M_{\theta}$ - Circumferential (hoop) bending moment

$m$ - Number of nodal points in shell element

$\mathrm{N}_{\mathrm{s}}$ - Meridional stress resultant

$\overline{\mathrm{N}}_{\mathbf{S}}$ - Applied meridional stress resultant

$\mathrm{N}_{\theta}$ - Circumferential (hoop) stress resultant

N - Matrix of stress resultants and bending moments

P - Potential energy functional

p - Pressure on shell

Q - Shear stress resultant

vi 


$$
\begin{aligned}
& \text { Q } \\
& R \quad \text { - Applied shear stress resultant } \\
& r \quad \text { - Radius of curvature } \\
& s \quad-\text { Shear on shell } \\
& s \quad-\text { Meridional coordinate } \\
& u \quad-\text { Shell strain energy functional } \\
& u_{n}-\text { Normal displacement } \\
& u_{r}-\text { Radial displacement } \\
& u_{s}-\text { Meridional displacement } \\
& u_{2}-\text { Axial displacement } \\
& z-\text { Axial coordinate }
\end{aligned}
$$

\section{SUBSCR IPTS}

i - Initial membrane stress or bending moment Also used as an index counter

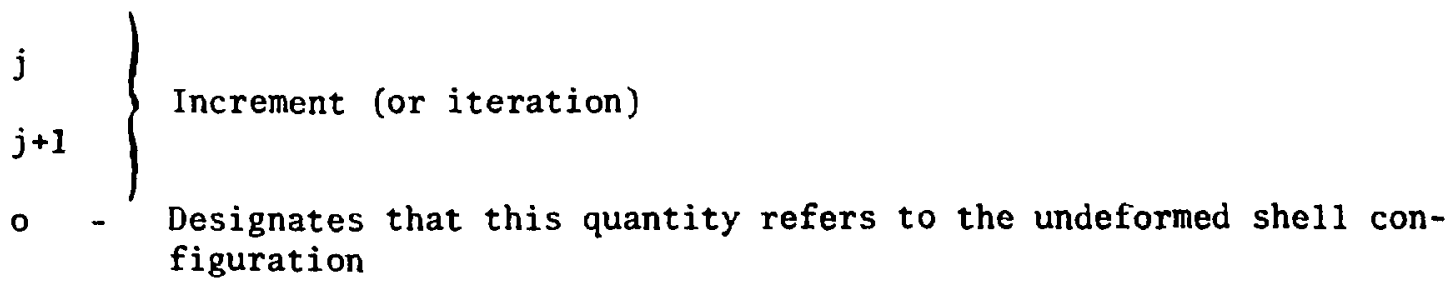
figuration

\section{SUPERSCRIPTS}

2,3 , and $m$ - The number of nodal points per element. Identifies the shape function to be used (see Appendix E).

GREEK

B - Rotation

$\bar{B}$ - Applied rotation

$\delta$ - Variation operator

$\delta_{i k}$ - Kronecker delta

$\Delta \quad$ - Incremental operator 


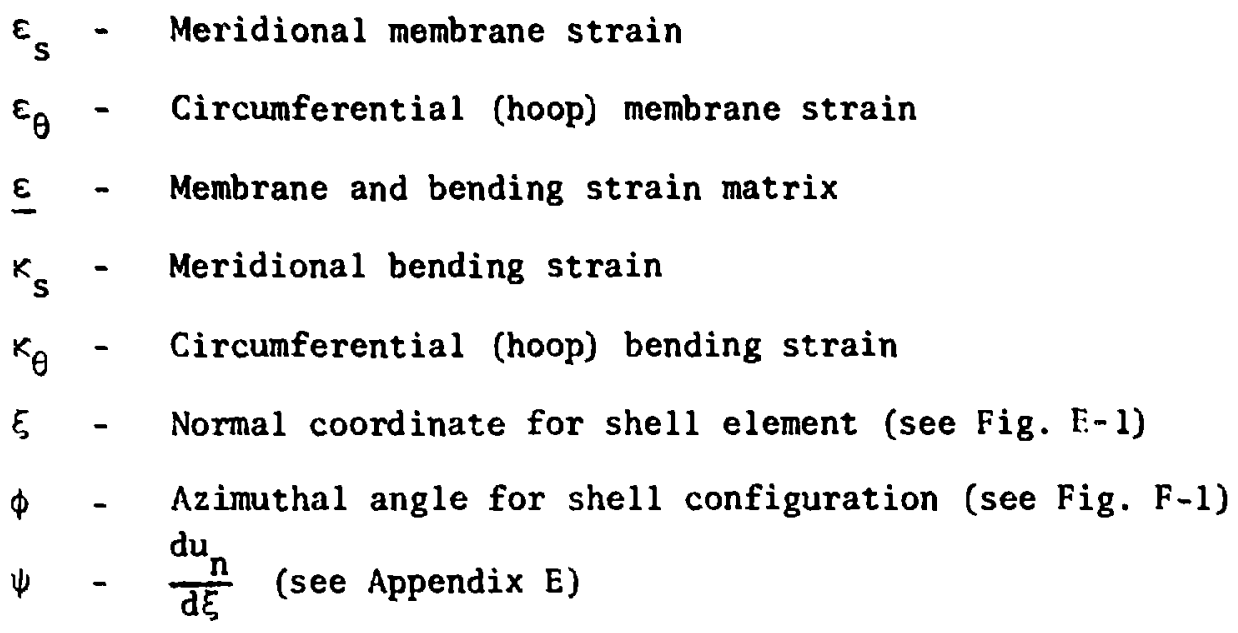

viii 
LINEAR AND NONLINEAR SYMMETRICALLY LOADED SHELLS OF REVOLUTION

APPROXIMATED WITH THE FINITE ELLMENT METHOD

by

William A. Cook

ABSTRACT

The development of a finite element nonlinear shell model is described. The approach uses incremental loads and a linear shell model with equilibrium iterations. Sixteen linear models are developed, eight using the potential energy variational principle and eight using a mixed variational principle. Four of these are suitable for extension to nonlinear shell theory. A nonlinear shell theory is derived, and a computational technique used in its solution is presented.

\section{INTRODUCTION AND RESULTS}

This report describes the development of a finite element nonlinear shell model for symmetrically loaded shells of revolution. This shell model is to be used with finite element continuum models as they exist in the NONSAP code. ${ }^{1,2}$ NONSAP has four- to eight-node axisymmetric isoparametric elements. To have a compatible number of common nodes, the shell model must have either two- or three-node elements. NONSAP solves nonlinear continuum problems by incrementing loads and then iterating linear continuum models to satisfy equilibrium.

Similarly, linear shell models must be studied when developing a nonlinear shell model.

In 1963 Grafton and Strome reported on the use of finite elements in the solution of symmetrically loaded shells. ${ }^{3}$ They introduced the two-node conical element, a very reliable element that is still used in many codes. Many researchers wanted a curved element, because many shells of revolution are spherical or elliptical in shape. Nickell and Sato reported on a two-node curved element in $1970 .{ }^{4}$ One of the objections to these elements is that the displacement function assumed for the shell is of higher order than the displacement 
functions assumed for continuum clements. Thus, when these elements adjoin one another, they are incompatible. To overcome this difficulty, Herrman, Taylor, and Green introduced a two-node conical element that was compatible with linear displacement continuum elenents. ${ }^{5}$ A mixed variational principle was used to accomplish this. Both the Grafton and Strome and the Nickell and Sato elements use tivo displacements and a meridional rotation as the generalized variables in the finite element approach. Herrman, Taylor, and Green use two displacements and the meridiona! moment.

The following linear shell models were developed (summarized in Table I).

- Potential Energy Model - Linear meridional displacement, cubic normal displacement, and quadratic rotation approximations.

1. Two-node conical element.

2. Two-noce curved element with curvature data required.

- Potential Energy Model - Quadratic meridional displacement, quintic normal displacoment, and quadratic rotation approximations.

3. Three-node curved element with curvature data required.

4. Three-node curved element with curvature data calculated from the three nodes.

- Mixed Model - Linear approximations for meridional displacement, normal displacement, and meridional moment.

5. Two-node conical element.

6. Two-node curved element with curvature data required.

- Mixed Model - Quadratic approximations for meridional jisplacement, normal displacement, and meridional moment.

7. Three-node curved element with curvature data required.

8. Three-node curved element with curvature data calculated from the three nodes.

- Potential Energy Model - Linear meridional displacement, cubic normal displacement, and quadratic rotation approximations. Also, for the hoop strain, a linear approximation for the radial displacement.

9. Two-node conical element.

10. Two-node curved elemert with curvature data required.

- Potential Energy Model - Quadratic meridional displacement, quintic normal displacement, and quartic rotation approximation. Also, for the hoop strain, a quadratic approximation for the radial displacement. 
11. Three-node curved element with curvature data required.

12. Three-node curved element with curvature data calculated from the three nodes.

- Mixed Model - Linear approximation for meridional displacement, and meridional inoment. Also, for the hoop strain, a linear approximation for the radial displacement.

13. Two-node conical element.

14. Two-node curved element with curvature data required.

- Mixed Model - Quadratic approximations for meridional displacement, normal displacement, and meridional moment. Also, for hoop strain, a quadratic approximation for the radial displacement.

15. Three-node curved element with curvature data required.

16. Three-node curved element with curvature data calculated from the three nodes.

The difference between the first eight models and the last eight models is the way the hoop strain is approximated. This results in different $B$ matrices (strain displacement approximations), which are described in Appendixes $A$ and $B$. The first eight models are simpler from a theoretical basis. However, the last eight models are simpler from a programming and calculational basis.

Several good references, such as Ref. 6, discuss the finite element method and how stiffness matrices and force vectors are accumulated. In this report the discussion of finite element formulations will be restricted to single element stiffnesses and force vector modifications caused by initial stress components. Models $1-4$ and 9-12 are derived in Sec. II, and models 5-8 and 13-16 are derived in Sec. III.

To evaluate these shell models, several problems were analyzed. First, Ref. 3 gives three example problems: a plate with a shear load, a cylindrical shell with a shear load, and a hemishperical shell with an applied moment. The nodal spacing used is shown in Ref. 3 , and shell models $1,4,5,8,9,12,13$, and 16 all solved these problems very accurately. The remaining shell models all require additional geometric data and are more accurate models because continuities and discontinuities can be modeled exactly between elements. Second, a hemisphere with pressure loading was analyzed using a mesh with coarse nodal spacing. The pressure loads were calculated to be compatible with two- and three-node continuum isoparametric element boundaries, thus making it possible to evaluate the response of the shell with a continuum. Shell models 1-4 and 
LINEAR FINITE ELEMENT SHELL MODELS

\begin{tabular}{|c|c|c|c|c|}
\hline Nodel & Formulation & $\begin{array}{l}\text { Nodes per } \\
\text { Elcaent }\end{array}$ & $\begin{array}{l}\text { Curvature } \\
\text { Required Input }\end{array}$ & $\begin{array}{l}\text { Radizl Disp. } \\
\text { Assumption }\end{array}$ \\
\hline 1 & P. E. & 2 & No & No \\
\hline 2 & & 2 & Yes & \\
\hline 3 & & 3 & res & \\
\hline 4 & $f$ & 3 & No & \\
\hline 5 & Mixed & 2 & No & \\
\hline 6 & & 2 & Yes & \\
\hline 7 & & 3 & Yes & \\
\hline 8 & & 3 & No & \\
\hline 9 & P. F. & 2 & No & Yes \\
\hline 10 & & 2 & Yes & \\
\hline 11 & & 3 & Yos & \\
\hline 12 & & 3 & No & \\
\hline 13 & Mixed & 2 & No & \\
\hline 14 & & 2 & Yes & \\
\hline 15 & & 3 & Yes & \\
\hline 16 & $t$ & 3 & No & $i$ \\
\hline
\end{tabular}

9-12 (potential energy formulation) are incompatible with the pressure loads, and the normal displacements oscillate about the exact result. The mixed formulation shell models 5-8 and 13-16 are compatible with the pressure loads and are more accurate. In summary, 88 example problems have been studied and are bound for reference.* The conclusion is that the mixed formulations with three nodes and curvature data supplied are the most accurate (shell models 7 and 15). However, all shell models are satisfactory if enough elements are used. The mixed variational principle, as presented in Appendix $C$, is restricted to small rotation theory. The models requiring that curvature data be supplied are not acceptable for nonlinear models that will be fincremented because the results of one calculation will alter the curvature of a following calculation. Thus, the shell models to be used for nonlinear shell analysis are 1, 4, 9, and 12 . Though these models were very accurate, they were the least accurate of the 16 shell models developed.

*Available from author on request. 
The computational technique used in Sec. IV to develop a nonlinear shell model consists of the following five steps.

1. Use a variational principle that satisfies equilibrium. This report uses the one described in Appendix $D$. This potential energy functional is a Eulerian formulation where the geometric quantities, the shell quintities, and the loads all refer to the deformed configuration of the shell.

2. Modify the variational principle for incremental loadings.

3. Linearize the variational principle such that it may be solved directly.

4. Use the finite element method to approximate the linearized incremental variational principle. This step is identical to solving a linear problem.

5. By iteration approximate the original nonlinear variational principle. This iteration is necossary because the variational principle was Iinearized, and itcration ensures that equilibrium is satisfied. The iteration step is identical to the increment step, except the applied loads do not change. Also, this iteration step makes it possible to include nonlinear materials.

To evaluate the nonlinear shell models $(1,4,9$, and 12), several problems were analyzed. First, the plate, the cylindrical, and the hemispherical problems described in Ref. 3 were analyzed in the nonlinear mode. Second, a cylindrical problem was analyzed with a large axial load. The axial displacement at the point of the load was nine times the length of the original shell. Third, a hemisphere with an internal pressure was analyzed. This pressure was large enough that the radial displacement was as large as the original radial coordinate. These models solved these problems accurately, and the solutions are bound for reference. Fourth, a cylindrical shell was loaded such that it would deform into a spherical shape. These nowlinear shell models would not solve this problem since the shape functions rectrict shell motion to small rotation.

II. LINEAR SHELLS USING POTENTIAL ENERGY VARIATIONAL PRINCIPLE

The potential energy functional described in Eqs. $(D-1)$ and $(D-2)$ can be written as

$$
\delta P\left(u_{s}, u_{n}, \beta\right)=\delta U\left(u_{n}, u_{n}, \beta\right)-\int_{L} r\left\{s \delta u_{s}+p \delta u_{n}\right\} d s
$$




$$
-r\left[\bar{N}_{s} \delta u_{s}+\bar{Q} \dot{\delta} u_{n}+\bar{M}_{s} \delta B\right]_{B},
$$

where

$$
\begin{aligned}
\delta U\left(u_{s}, u_{n}, B\right)= & \int_{i} r\left\{N_{s} \delta \varepsilon_{s}+N_{\theta} \delta \varepsilon_{\theta}+M_{s} \delta k_{s}+M_{\theta} \delta K_{\theta}\right. \\
& \left.+Q\left(\delta \beta-\frac{\delta u_{s}}{R}+\frac{d\left(\delta u_{n}\right)}{d s}\right)\right\} d s .
\end{aligned}
$$

The strains $\varepsilon_{5}, \varepsilon_{\theta}, K_{s}, k_{\theta}$ arc defined as

$$
\begin{aligned}
& \varepsilon_{s}=\frac{d u_{s}}{d s}+\frac{u_{n}}{R}, \\
& \varepsilon_{\theta}=\frac{\cos \phi u_{s}-\sin \phi u_{n}}{r}=\frac{u_{r}}{r}, \\
& \left.\begin{array}{l}
K_{s}=\frac{d \beta}{d s}, \text { and } \\
k_{\theta}=\frac{\beta \cos \phi}{r}
\end{array}\right\} \\
& \text { where } \beta=\frac{u_{s}}{R}-\frac{d u_{n}}{d s} \text {. }
\end{aligned}
$$

For constitutive relations we used the following,

$$
N_{s}=C_{s} \varepsilon_{s}+C_{s \theta} \varepsilon_{\theta}+N_{s i}
$$




$$
\begin{aligned}
& N_{\theta}=C_{s \theta} \varepsilon_{s}+C_{\theta} \varepsilon_{\theta}+N_{\theta i} \\
& M_{s}=D_{s} k_{s}+D_{s} k_{\theta}+M_{s i} \\
& M_{\theta}=D_{s \theta^{k}}+D_{\theta} \kappa_{\theta}+M_{\theta i} \text {, } \\
& \delta U\left(u_{s}, u_{n}, B\right)=\int_{i} r\left\{\left(C_{s} \varepsilon_{s}+C_{s} \varepsilon_{\theta} \varepsilon\right) \delta \varepsilon_{s}+\left(C_{s} \varepsilon_{s}+C_{\theta} \varepsilon_{\theta}\right) \delta \varepsilon_{\theta}\right. \\
& \left.+\left(D_{s} k_{s}+D_{s} k_{\theta}\right) \delta k_{s}+\left(D_{s} k_{s}+D_{\theta} k_{\theta}\right) \delta k_{\theta}\right\} d s \\
& +\int r\left\{N_{s i} \delta \varepsilon_{s}+N_{\theta i} \delta \varepsilon_{\theta}+M_{s i} \delta k_{s}+M_{\theta i} \delta \kappa_{\theta}\right\} d s \\
& =\int_{\mathrm{L}}^{r} \underline{\varepsilon}^{\mathrm{T}} \underline{\underline{0}} \delta \underline{\varepsilon} \mathrm{d} s+\int_{\mathrm{L}} \mathrm{r} \underline{\mathrm{N}}_{-\mathrm{i}}^{\mathrm{T}} \delta \underline{\varepsilon} \mathrm{ds} \text {, }
\end{aligned}
$$

where

$$
\begin{aligned}
& \underline{\varepsilon}^{\mathrm{T}} \equiv\left(\varepsilon_{s} \varepsilon_{\theta} \kappa_{s} \kappa_{\theta}\right) \\
& \underline{N}^{\mathrm{T}} \equiv\left(N_{s i} N_{\theta i} M_{s i} M_{\theta i}\right)
\end{aligned}
$$

and

$$
\underline{D}=\left[\begin{array}{cccc}
C_{s} & C_{s \theta} & 0 & 0 \\
C_{s \theta} & C_{\theta} & 0 & 0 \\
0 & 0 & D_{s} & D_{s \theta} \\
0 & 0 & D_{s \theta} & D_{\theta}
\end{array}\right] .
$$

7 


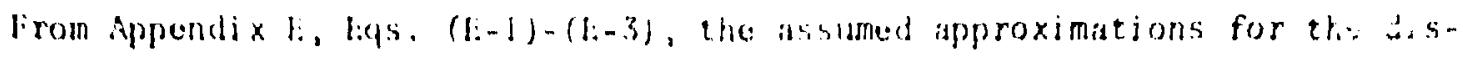
placements and rotiations over cach element are

$$
\begin{aligned}
& u_{s}(s)=\sum_{i=1}^{m} h_{s i}^{m} u_{s i} \\
& u_{n}(E)=\sum_{i=1}^{m}\left\{h_{B i}^{m} q_{i} c_{i} u_{s i}+h_{n i}^{m} u_{n i}-h_{E i}^{m} q_{i} \beta_{i}\right\} \text {, and } \\
& B\left(s_{2}\right)=\sum_{i=1}^{m}\left\{\left[c(\xi) h_{s i}^{\mathrm{m}}-\frac{q_{i} c_{i}}{q(\xi,)}-\frac{d h_{f i}^{m}}{d \xi}\right] u_{s i}-\frac{1}{q(\xi)} \frac{d h_{n i}^{m}}{d \xi} u_{n i}\right. \\
& \left.+\frac{q_{i}}{q\left(\varepsilon_{j}\right)}-\frac{d h_{\beta i}^{m}}{d \varepsilon_{2}} \beta_{i}\right\}
\end{aligned}
$$

When these assumed displacements and rotations are used to approximate the strain $E_{s}, \varepsilon_{\theta}, K_{s}$, and $K_{0}$.

$$
\begin{aligned}
\varepsilon_{s}=\frac{d u_{s}}{d s}+\frac{u_{n}}{R} & =\sum_{i=1}^{m}\left\{\left[\frac{1}{q(\xi)} \frac{d h_{s i}^{m}}{d \xi}+q_{i} c_{i} h_{B i}^{m} c(\xi)\right] u_{s i}\right. \\
& \left.+c(\xi) h_{n i}^{m} u_{n i}-c(\xi) h_{B i}^{m} q_{i} \beta_{i}\right\} ; \\
\epsilon_{\theta}=\frac{u_{s} \cos \phi}{+}+u_{n} \sin \phi & r \\
r & \sum_{i=1}^{m}\left\{\left[\frac{\cos \phi(\xi)}{r(\xi)} h_{s i}^{m}+\frac{\sin \phi(\xi)}{r(\xi)} h_{B i}^{m} q_{i} c_{i}\right] u_{s i}\right. \\
& \left.+\frac{\sin \phi(\xi)}{r(\xi)} h_{n i}^{m} u_{n i}-\frac{\sin \phi(\xi)}{r(\xi)} q_{i} h_{B i}^{m} \beta_{i}\right\},
\end{aligned}
$$




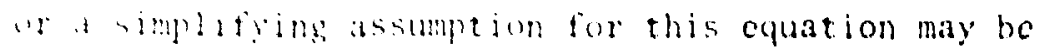

$$
\begin{aligned}
& u_{r}(\xi)-\sum_{i=1}^{m} h_{s i}^{m} u_{r i} \text { and then } \varepsilon_{\theta}=\sum_{i=1}^{m} \frac{h_{s i}^{m}}{r(\xi)} u_{r i} \text {; } \\
& k_{s}=\frac{d \beta}{d s}=\sum_{i=1}^{m} \int\left[\frac{h_{s i}^{m !}}{q(5)} \frac{d c(\xi)}{d \xi}+\frac{c(\xi)}{q(\xi)} \frac{d h_{s i}^{m}}{d \xi}-\frac{q_{i} c_{i}}{q(\xi)} \frac{d h_{B i}^{m}}{d \xi} \frac{d}{d \xi}\left(\frac{1}{q(\xi)}\right)\right. \\
& \left.-\frac{4_{i}}{(q(\xi))} \frac{d^{2} h_{R i}^{m}}{d !}\right] u_{s i}-\left[\frac{1}{q(\xi,)} \frac{d h_{n i}^{m}}{d \xi} \frac{d}{d \xi}\left(\frac{1}{q(\xi)}\right)+\frac{1}{(q(\xi))^{2}}\right. \\
& \left.\frac{d^{2} n_{n i}^{m}}{d \xi^{2}}\right] n_{n i}+\left[\frac{q_{i}}{q(\xi)} \cdot \frac{d h_{B i}^{m}}{d \xi} \frac{d}{d \xi}\left(\frac{1}{q(\xi)}\right)+\frac{q_{i}}{(q(\xi))^{2}} \frac{d^{2} h_{B i}^{m}}{d \xi^{2}}\right] B_{i} \mid \text {; and } \\
& \kappa_{\theta}=\frac{B \cos \phi}{r}=\sum_{i=I}^{m}\left\{\left[\left(c(\xi) h_{s i}^{m}-\frac{q_{i} c_{i}}{q(\xi)} \frac{d h_{B i}^{m}}{d \xi}\right) \frac{\cos \phi(\xi)}{r(\xi)}\right] u_{s i}\right. \\
& \left.-\left[\frac{d h_{n i}^{m}}{d \xi} \frac{\cos \phi(\xi)}{q(\xi) r(\xi)}\right] u_{n i}+\left[\frac{q_{i}}{q(\xi)} \frac{d_{B i}^{m}}{d \xi} \frac{\cos \phi(\xi)}{r(\xi)}\right] B_{i}\right\}
\end{aligned}
$$

At this point transform the displacements from $u_{s i}, u_{n i}$ to $u_{r i}, u_{z i}$. This is accomplished with the following transformation equations,

$$
\begin{aligned}
& u_{s i}=\cos \phi_{i} u_{r i}-\sin \phi_{i} u_{z i} \text { and } \\
& u_{n i}=\sin \phi_{i} u_{r i}+\cos \phi_{i} u_{z i}
\end{aligned}
$$

where $\phi_{i}=\left(\bar{\xi}_{i}\right), \xi_{i}$ being the value of $\xi$ at nodal point $i$. 
The relation between the stralns and the displacements la taken to be

$$
\text { E. }=\underline{B} \mathrm{u}
$$

where

$$
\begin{aligned}
& u^{q}=\left(u_{r l}, u_{2 l}, a_{1}, \ldots ., a_{m}\right) \text { and }
\end{aligned}
$$

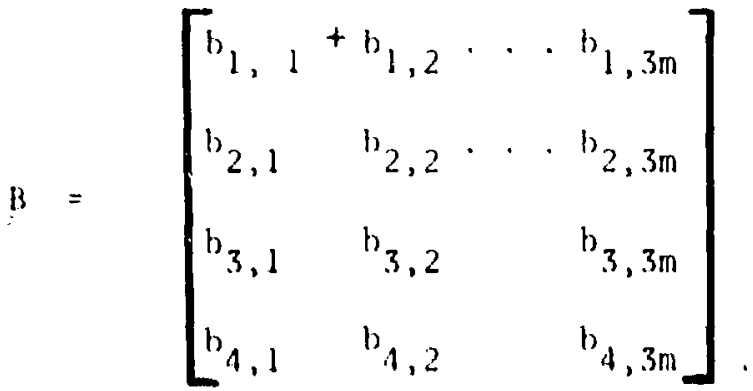

The $b_{i, j}$ terms are tabulated in Appendix $\Lambda$.

When liq. (10) is substituted into I:q. (5), we obtain

$$
\delta u=u^{T} \int_{L_{e}} r B^{T} \| B d s \delta \underline{u}+\int_{L_{c}} r \underline{N}_{-} B d s \delta \underline{u} .
$$

Thus the stiffness matrix is

$$
\int_{-1}^{1} \mathrm{r}(\xi) \mathrm{q}(\xi) \stackrel{\mathrm{B}^{\mathrm{T}}}{\underline{\mathrm{D}}} \underline{\underline{\mathrm{B}} \mathrm{d} \xi},
$$

because $d s=q(\xi) d \xi$, and the modification to the force vector due to initial stress is

$$
\int_{-1}^{1} r(\xi) q(\xi) \underline{N}_{i}^{\mathrm{T}} \stackrel{\mathrm{B}}{=} \mathrm{d} \xi
$$


The resulting stifness matrix equation approximates the equilibrjum cquations in Aprendix 1 , lquations (12) and (1.3) can he ealculated numerically provided the following quantities are hnown:

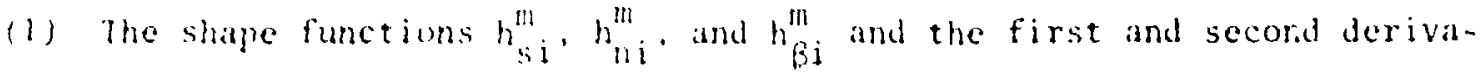
tives of the shaje functions with respect to 5 , (see Appendix E).

(:) The radial and axial coordinates $r(s),, z(5)$.

(i) The nomalized meridional length $q(\xi)$ and $q_{j} \equiv q\left(\xi_{i}\right)$. Also the derivative $\frac{d}{d}\left(\frac{1}{4 !}\right)$,

(:) the azimuthal angle $+i s_{1}$ and $t_{j}=\left(_{i}\right)$, and

(5) The curvature $c(s)=\frac{1}{R(i)}$ where $s_{i}=c\left(s_{-j}\right)$, and also, the derivative dis $\frac{1}{d !}$

iwo approaches aro descrihed in Mpendix (; for detormining items (2) through (5).

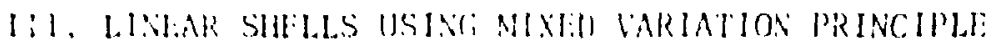

The functional described by liqs. $(C-1-(-3)$ can be written as

$$
\begin{aligned}
\delta I\left(u_{s}, u_{n}, M_{s}\right) & =\delta U\left(u_{s}, u_{n}, M_{s}\right)-\int_{L} r\left\{s \delta u_{s}+p \delta u_{n}\right\} d s \\
& -r\left[\bar{N}_{s} \delta u_{s}+\bar{Q} \delta u_{n}+\bar{B} \delta M_{s}\right]_{B},
\end{aligned}
$$

where

$$
\begin{aligned}
\delta U\left(u_{s}, u_{n}, M_{s}\right) & =\int\left\{r N_{s} \delta \varepsilon_{s}+r N_{\theta} \delta \varepsilon_{\theta}-r\left[\frac{M_{s}}{D_{s}}+\left(1-\frac{D_{s}}{D_{s}}\right) \kappa_{\theta}\right] \delta M_{s}\right. \\
& -r \beta \frac{d\left(\delta M_{s}\right)}{d s}-r \quad Q\left(M_{s}, k_{\theta}\right)\left[\frac{\delta u_{s}}{R}-\frac{d\left(\delta u_{n}\right)}{d s}\right] d s
\end{aligned}
$$

and 


$$
Q\left(M_{s}, K_{\theta}\right)=\frac{d M_{s}}{d s}+\left(1-\frac{D_{s \theta}}{D_{s}}\right) \frac{\cos \phi}{r} M_{s}-\left(D_{\theta}-\frac{D_{s \theta}^{2}}{D_{s}}\right) k_{\theta} \frac{\cos \phi}{r} .
$$

The strains $\varepsilon_{s}, \varepsilon_{\theta}$, and $\kappa_{\theta}$ are

$$
\begin{aligned}
& \varepsilon_{s}=\frac{d u_{s}}{d s}+\frac{u_{n}}{R}, \\
& \varepsilon_{\theta}=\frac{\cos \phi u_{s}+\sin \phi u_{n}}{r}=\frac{u_{r}}{r}, \text { and } \\
& \kappa_{\theta}=\beta\left(u_{s}, u_{n}\right) \frac{\cos \phi}{r}=\left[\frac{u_{s}}{R}-\frac{d u_{n}}{d s}\right] \frac{\cos \phi}{r} .
\end{aligned}
$$

For constitutive relations, we have

$$
\begin{aligned}
& N_{s}=C_{s} \varepsilon_{s}+C_{s \theta} \varepsilon_{\theta}+N_{s i} \\
& N_{\theta}=C_{s \theta} \varepsilon_{s}+C_{\theta} \varepsilon_{\theta}+N_{\theta i} \\
& \left.\begin{array}{l}
M_{\theta}=D_{s} K_{s}+D_{s \theta} K_{\theta} \\
M_{s}=D_{s \theta} K_{s}+D_{\theta} K_{\theta}
\end{array}\right\} \text { where } k_{s}=\frac{d B\left(u_{s}, u_{n}\right)}{d s},
\end{aligned}
$$

Eq. (15) can be written as

$$
\delta U\left(u_{s}, u_{n}, M_{s}\right)=\int_{L} r \underline{\varepsilon}^{T} \underline{D}=\delta \underline{\varepsilon} d s+\int_{L} r \underline{N}_{i}^{T} \underline{D}=\delta \underline{\varepsilon} d s,
$$


where

$$
\underline{E}^{T}=\left(\varepsilon_{s}, \varepsilon_{\theta}, M_{s}, K_{\theta}, \frac{d M_{s}}{d s} B\left(u_{s}, u_{n}\right)\right)
$$

$D=\left(\begin{array}{cccccc}C_{s} & C_{s \theta} & 0 & 0 & 0 & 0 \\ C_{s \theta} & C_{\theta} & 0 & 0 & 0 & 0 \\ 0 & 0 & -1 & -1+\frac{D}{D} \theta & 0 & 0 \\ 0 & 0 & -1+\frac{D_{s}}{D} D_{s} & D_{\theta}-\frac{D_{s}}{D_{s}} & 0 & 0 \\ 0 & 0 & 0 & 0 & 0 & -1 \\ 0 & 0 & 0 & 0 & -1 & 0\end{array}\right)$

$\underline{N}_{i}^{T}=\left(N_{s i}, N_{\theta i}, 0,0\right)$.

Let both displacements and the moment be approximated over each element with the same shape functions. The displacements and moments are given by

$$
\begin{aligned}
& u_{s}(\xi)=\sum_{i=1}^{m} h_{s i}^{m} u_{s i} \\
& u_{n}(\xi)=\sum_{i=1}^{m} h_{s i}^{m} u_{\cdot i i} \\
& M_{s}(\xi)=\sum_{i=1}^{m} h_{s i}^{m} M_{s i}
\end{aligned}
$$


When these assumed displacements are substituted into the $\underline{\varepsilon}$ quantities one gets

$$
\begin{aligned}
& \varepsilon_{s}=\sum_{i=1}^{m}\left\{\frac{1}{q(\xi)} \frac{d h_{s i}^{m}}{d \xi} u_{s i}+c(\xi) h_{s i}^{m} u_{n i}\right\} ; \text { and } \\
& \varepsilon_{\theta}=\sum_{i=1}^{m}\left\{h_{s i}^{m} \frac{\cos \phi(\xi)}{r(\xi)} u_{s i}+h_{s i}^{m} \frac{\sin \phi(\xi)}{r(\xi)} u_{n i}\right\} \\
& \text { or, assuming } u_{r}(\xi)=\sum_{i=1}^{m} h_{s i}^{m} u_{r i} \text {, } \\
& \varepsilon_{\theta}=\sum_{i=1}^{m} \frac{h_{s i}^{m}}{r(\varepsilon)} u_{r i} \\
& M_{s}=\sum_{i=1}^{m} h_{s i}^{m} M_{s i} \\
& \kappa_{\theta}=\sum_{i=1}^{m}\left\{c(\xi) h_{s i}^{m} \frac{\cos \phi(\xi)}{r(\xi)} u_{s i}-\frac{\cos \phi(\xi)}{q(\xi) r(\xi)} \frac{d h_{s i}^{m}}{d \xi} u_{n i}\right\} ; \\
& \frac{d M_{s}}{d s}=\sum_{i=1}^{m} \frac{1}{q(\xi)} \frac{d h_{s i}^{m}}{d \xi} M_{s i} ; \text { and } \\
& B\left(u_{s}, u_{n}\right)=\sum_{i=1}^{m}\left\{c(\xi) n_{s i}^{m} u_{s i}-\frac{1}{q(\xi)} \frac{d h_{s i}^{m}}{d \xi} u_{n i}\right\} .
\end{aligned}
$$


At this point transform the displacements $u_{i}, u_{n i}$, to $u_{r i}, u_{2 i}$. This is accomplished with the following transformation equations:

$$
\begin{aligned}
& u_{s i}=\cos \phi_{i} u_{r i}-\sin \phi_{i} u_{z i} \text { and } \\
& u_{n i}=\sin \phi_{i} u_{r i}+\cos \phi_{i} u z i
\end{aligned}
$$

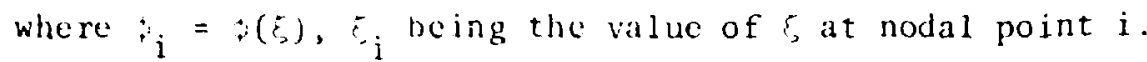
Thus

$$
\underline{\underline{\varepsilon}}=\underline{B} \underline{\underline{u}}
$$

where

$$
\underline{u}^{\mathrm{I}}=\left(u_{r 1}, u_{21}, \mathrm{u}_{s 1} \cdot \ldots \mathrm{M}_{s \mathrm{~m}}\right)
$$

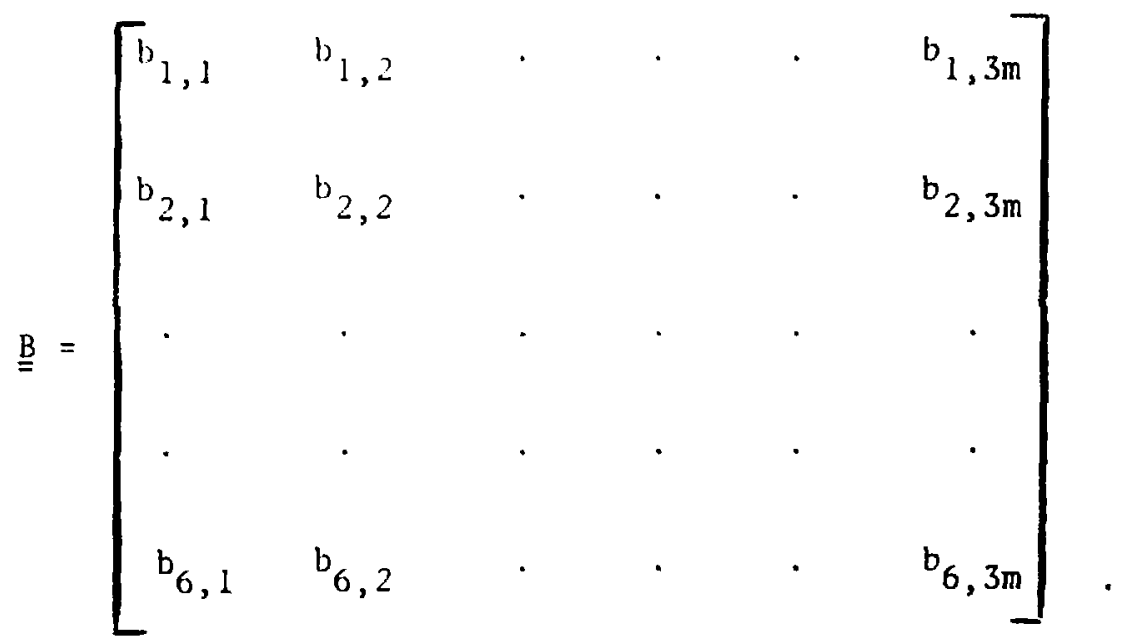

The $b_{i, j}$ terms are tabulated in Appendix $B$. When Eq. (24) is substituted into Eq. (19), one obtains

$$
\delta U=\underline{u}^{T} \int_{L_{e}} r \stackrel{B^{T}}{=} \stackrel{D}{=} \stackrel{B}{=} d: \quad \delta \underline{u}+\int_{L_{e}} r \underline{N}_{i}^{T} \stackrel{B}{=} d s \delta \underline{u} .
$$

Thus this element stiffness matrix is 


$$
\int_{-1}^{1} r(\xi) q(\xi) \underline{\underline{B}}^{\mathrm{T}} \stackrel{\underline{D}}{=} \mathrm{d} \xi
$$

since $d s=q(\xi) d \xi$

and the modification to the force vector from initial stresses is

$$
\int_{-1}^{1} r(\xi) q(\xi) \stackrel{N_{-}^{T}}{\mathrm{~B}} \mathrm{~d} d \xi .
$$

Equations (26) and (27) can be calculated numerically provided the following quantities are known:

(1) the shape functions $h_{s i}^{m}$ and the first derivative of the shape function $\frac{\mathrm{dh}_{\mathrm{si}}^{\mathrm{m}}}{\mathrm{d} \xi}$ (see Appendix E),

(2) the radial and axial coordinates $r(\xi)$ and $z(\xi)$,

(3) the normalized meridional length $q(\xi)$,

(4) the azimuthal angle $\phi(\xi)$ and $\phi_{i} \equiv \phi\left(\xi_{i}\right)$, and

(5) the curvature $\mathrm{c}(\xi) \equiv \frac{1}{\mathrm{R}(\xi)}$.

Two approaches are described in Appendix $G$ for determining items (2) through (5).

IV. NONLINEAR SHELLS USING POTENTIAL ENERGY VARIATIONAL PRINCIPLE

The derivation of the shell equilibrium equations in Appendix $F$ and the potential energy functional in Appendix $D$ are both valid for large strain conditions when the geometric quantities $r, z, \phi, s, R, L$, and $B$; the shell quantities $N_{S}, N_{\theta}, M_{S}$, and $M_{\theta}$; and the loads per area $p$ and $S$ all refer to the deformed configuration of the shell. However, the shape functions from Appendix E limit this approach to small rotations. Thus

$$
\begin{aligned}
& \delta P\left(u_{s}, u_{n}, \beta\right)=U\left(u_{s}, u_{n}, \beta\right)-\int_{L} r\left\{s \delta u_{s}+p \delta u_{n}\right\} d s \\
& -r\left[\bar{N}_{s} \delta u_{s}+\bar{Q} \delta u_{n}+\bar{M}_{s} \delta \beta\right]_{B}=0
\end{aligned}
$$


where

$$
\begin{aligned}
\delta u\left(u_{s}, u_{n}, B\right) & =\int_{L} r N_{s}\left(\frac{d\left(\delta u_{s}\right)}{d s}+\frac{\delta u_{n}}{R}\right)+N_{\theta} \frac{\delta u_{r}}{r} \\
& \left.+r_{s} \frac{d(\delta \beta)}{d s}+M_{\theta} \frac{\cos \phi}{r} \delta \beta+Q\left(\frac{d\left(\delta u_{n}\right)}{d s}+s \beta-\frac{\delta u_{s}}{R}\right)\right\} d s .
\end{aligned}
$$

The membrane and bending strains associated with nonlinear shells are defined in Appondix $H$.

To increment for a nonlinear solution, consider Eqs. (28) and (29) for the $j+1$ increment. The variation of the potential energy functional is

$$
\begin{gathered}
\delta U\left(u_{s_{j+1}}, u_{n_{j+1}}, \beta_{j+1}\right)-\int_{L_{j+1}}\left\{s_{j+1} \delta u_{s_{j+1}}+p_{j+1} \delta u_{j+1}\right\} s_{j+1} \\
-r_{j+1}\left\{\hat{N}_{s_{j+1}} \delta u_{s_{j+1}}+\bar{Q}_{j+1} \delta u_{n_{j+1}}+\bar{M}_{s_{j+1}} \delta \beta_{j+1}\right\}_{B}=0
\end{gathered}
$$

where

$$
\begin{aligned}
& \delta u\left(u_{s_{j+1}}, u_{n_{j+1}}, \beta_{j+1}\right)=r_{j+1} \int_{L_{j+1}}\left(\frac{d\left(\delta u_{j+1} s_{j+1}\right.}{d s_{j+1}}+\frac{\delta u_{n_{j+1}}}{R_{j+1}}\right) \\
& +N_{\theta_{j+1}} \frac{{ }^{\delta r_{j+1}}}{r_{j+1}}+M_{s_{j+1}} \frac{d\left(\delta \beta_{j+1}\right)}{d s_{j+1}}+M_{\theta_{j+1}} \frac{\cos \phi_{j+1}}{r_{j+1}} \\
& \delta \beta_{j+1}+Q_{j+1}\left(\frac{d\left(\delta u_{j+1}\right)}{d s_{j+1}}+\delta \beta_{j+1}-\frac{\delta u_{s+1}}{R_{j+1}}\right) d s_{j+1}
\end{aligned}
$$




\section{Make the following assumptions.}

- The incremental displacements, rotations, membrane and bending strains, stress results, and bending moments are as described in Appendixes I and $\mathrm{J}$.

- The variations of displacements and rotation are

$$
\begin{aligned}
& \delta u_{s_{j+1}}=\delta\left(\Delta u_{s_{j}}\right), \\
& \delta u_{n_{j+1}}=\delta\left(\Delta u_{n_{j}}\right), \text { and } \\
& \delta B_{j+1}=\delta\left(\Delta \beta_{j}\right),
\end{aligned}
$$

- The following quantities are approximately equal

$$
\begin{aligned}
& R_{j+1} \simeq R_{j}, \\
& d s_{j+1} \simeq d s_{j}, \\
& L_{j+1} \simeq L_{j}, \\
& B_{j+1} \simeq B_{j}, \\
& r_{j+1} \simeq r_{j}, \text { and } \\
& \phi_{j+1} \simeq \phi_{j} .
\end{aligned}
$$

With these assumptions

$$
\delta U\left(\Delta u_{s_{j}}, \Delta u_{n_{j}}, \Delta \beta_{j}\right)-\int_{L_{j}} r_{j}\left\{s_{j+1} \delta\left(\Delta u_{s_{j}}\right)+p_{j+1} \delta\left(\Delta u_{n_{j}}\right\} d s_{j}\right.
$$




$$
-r_{j}\left\{\bar{N}_{s_{j+1}} \delta\left(\Delta u_{s_{j}}\right)+\bar{Q}_{j+1} \quad \delta\left(\Delta u_{n_{j}}\right)+\bar{M}_{s_{j+1}} \delta\left(\Delta B_{j}\right)\right\}_{B_{j}}=0
$$

or

$$
\delta U\left(\Delta u_{s_{j}}, \Delta u_{n_{j}}, \Delta \beta_{j}\right)=\int_{L_{j}} x_{j} \underline{\varepsilon}_{-j}^{T} \stackrel{D}{=} \delta \underline{\varepsilon}_{j} d s_{j}+\int_{L_{j}} r_{j}\left(N_{i}\right)_{j}^{T} \delta \underline{\varepsilon}_{j} d s_{j},
$$

where

$$
\begin{gathered}
\underline{\varepsilon}_{j}^{T} \equiv\left(\Delta \varepsilon_{s_{j}}, \Delta \varepsilon_{\theta_{j}}, \Delta k_{s_{j}}, \Delta k_{0}\right) \\
\left(\underline{N}_{i}\right)_{j}^{T} \equiv\left(N_{s_{j}}+\left(N_{s_{j}}\right)_{j+1}, N_{\theta_{j}}+\left(N_{\theta_{i}}\right)_{j+1}, M_{s_{j}}+\left(M_{s_{i}}\right)_{j+1},\right. \\
\left.M_{\theta_{j}}+\left(M_{\theta_{i}}\right)_{j+1}\right)
\end{gathered}
$$

$$
\underline{D}_{j} \equiv\left[\begin{array}{cccc}
c_{s_{j}} & c_{s \theta_{j}} & 0 & 0 \\
c_{s \theta_{j}} & c_{\theta_{j}} & 0 & 0 \\
0 & 0 & D_{s_{j}} & D_{s \theta_{j}} \\
0 & 0 & D_{s \theta_{j}} & D_{\theta_{j}}
\end{array}\right]
$$

19 
$\mathrm{Q}_{\mathrm{j}} \mathrm{is}$ the tangential material property matrix. Soc Appendix $K$ for detalls concerning nonlinear material properties.

liquations(32), (33), and (34) are identicial to liqs. (1), (5), and (6). Thus, the solution approach described in Section II applies to each increment and iteration for nonlinear shell models. Again, the itcration step is the same as the increment step except the applied loads do not change. These are

$$
\begin{aligned}
& S_{j+1}=s_{j}, \\
& p_{j+1}=p_{j} \\
& \bar{N}_{s_{j+1}}=\bar{N}_{s_{j}}, \\
& \bar{Q}_{j+1}=\bar{Q}_{j}, \\
& \bar{M}_{n+1}=\bar{M}_{s_{j}}, \\
& \left(\mathrm{~N}_{s_{i}}\right)_{j+1}=\left(\mathrm{N}_{s_{i}}\right)_{j}, \\
& \left(N_{\theta_{i}}\right)_{j+1}=\left(N_{\theta_{i}}\right)_{j}, \\
& \left(M_{s_{i}}\right)_{j+1}=\left(M_{s_{j}}\right)_{j} \text {, and } \\
& (M)_{j+1}=\left(M_{\theta_{i}}\right)_{j} \text {. }
\end{aligned}
$$

\section{RECOMMENDATIONS}

From this study several recommendations can be made.

1. Develop additional example problems to check out and improve the nonlinear shell models.

2. Study methods of improving the convergence criteria and the convergence rate for the nonlinear shell models.

3. Investigate variational methods, for example 
a. A Lagrangian (unleformed geometric configuration) approach, which would be compatible with the NokSA cot?.

b. A mixed formulation using either the lagrangian (undeformed geometric configuration) approach or the lulerian (deformed geometric configurationl approach.

With one of these variational methods better finite element shell models could be used for nonlinear analysis.

4. Develop an improved shell model by adding shear deformation and rotatory inertia. Also consider changes in shell thickness as the shell stretches or is compressed. Such changes would have to be consistent with the shell theory assumption.

5. Investigate other methods of solving the nonlinear equations hesides iterating.

6. Eliminate the small rotation restrictions. 
22

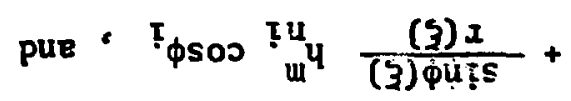

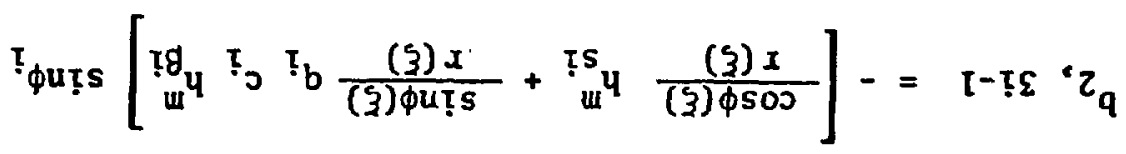

- $\tau_{\phi u t s} \stackrel{\text { tu }}{u}_{u} \frac{(\xi) x}{(\xi) \phi u t ̦ s}+$

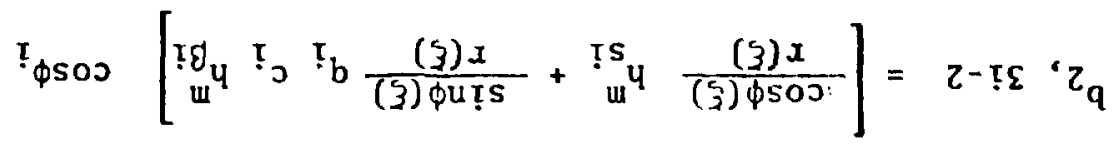

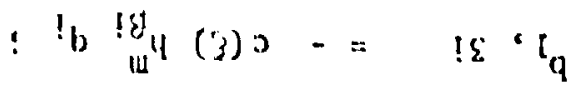

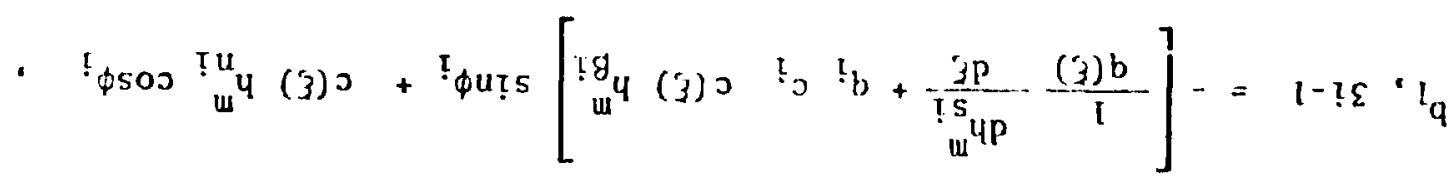

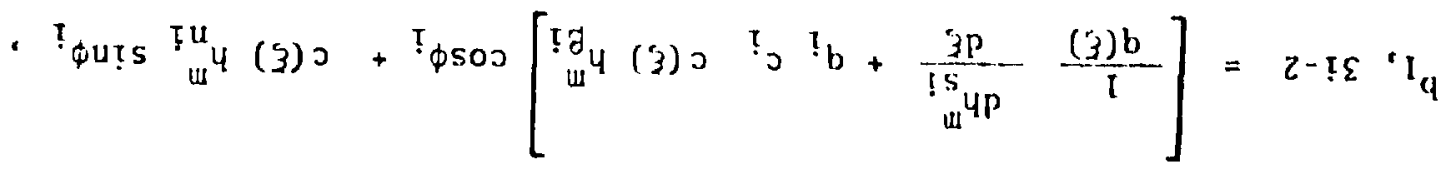

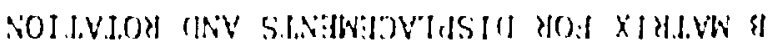

$V$ XIIN:HAN 


$$
b_{2,3 i}=-\frac{\sin (\varepsilon)}{r(\varepsilon)} q_{i} h_{B i}^{m} \text {, }
$$

Or, using the simplifying assumption $u_{r}(5)=\sum_{i=1}^{m} h_{s i}^{m}{ }_{r i}$,

$$
\begin{aligned}
& b_{2,3 i-2}=\frac{h_{5 i}^{m}}{r(\xi)}, \\
& b_{2,3 i-1}=0, \\
& b_{2,3 i}=0 ;
\end{aligned}
$$

$$
\begin{aligned}
b_{3,3 i-2} & =\left[\frac{h_{s i}^{n}}{q(\xi)} \frac{d c(\xi)}{d \xi}+\frac{c(\xi)}{q(\xi)} \frac{d h_{s i}^{m}}{d \xi}-\frac{q_{i} c_{i}}{q(\xi)} \frac{d h_{\beta j}^{m}}{d \xi} \frac{d}{d \xi}\left(\frac{1}{q(\xi)}\right)\right. \\
& \left.-\frac{q_{i} c_{i}}{(q(\xi))^{2}} \frac{d^{2} h_{B i}^{m}}{d \xi^{2}}\right] \cos \phi_{i}-\left[\frac{1}{q(\xi)} \frac{d h_{n i}^{m}}{d \xi} \frac{d}{d \xi}\left(\frac{1}{q(\xi)}\right)\right. \\
& \left.+\frac{1}{(q(\xi))^{2}} \frac{d^{2} h_{n i}^{m}}{d \xi^{2}}\right] \sin \phi_{i} .
\end{aligned}
$$




$$
\begin{aligned}
& b_{3,3 i-1}=-\left[\frac{h_{s i}^{m}}{q(\xi)} \frac{d c(\xi)}{d \xi}+\frac{c(\xi)}{q(\xi)} \frac{d h_{s i}^{m}}{d \xi}-\frac{q_{i} c_{i}}{q(\xi)} \cdot \frac{d h_{B i}^{m}}{d \xi} \frac{d}{d \xi}\left(\frac{1}{q(\xi)}\right)\right. \\
& \left.-\frac{q_{i} c_{i} d^{2} n_{\beta i}^{m}}{(q(\xi))^{2}} \cdot \frac{s^{2}}{d \xi^{2}}\right] \sin i-\left[\frac{1}{q(\xi)} \frac{d n_{n i}^{m}}{d \xi} \frac{d}{d \xi}\left(\frac{1}{q(\xi)}\right)\right. \\
& \left.+\frac{1}{(q(\xi))^{2}} \frac{d^{2} h_{n i}^{n}}{d \xi^{2}}\right] \cos \phi_{j} \\
& \mathrm{~b}_{3,3 i}=\frac{q_{\mathrm{i}}}{q(\xi)} \frac{d h_{\beta i}^{\mathrm{m}}}{\mathrm{d} \xi} \frac{\mathrm{d}}{\mathrm{d} \xi}\left(\frac{1}{q(\xi)}\right)+\frac{q_{i}}{(q(\xi))^{2}} \frac{\mathrm{d}^{2} h_{\beta i}^{m}}{d \xi^{2}} ; \\
& b_{4,3 i-2}=\left[c(\xi) h_{s i}^{m}-\frac{q_{i} c_{i}}{q(\xi)} \frac{d h_{B i}^{m}}{d \xi}\right] \frac{\cos \phi(\xi)}{r(\xi)} \cos \phi_{i} \\
& -\frac{d h_{n i}^{m}}{d \xi} \frac{\cos \phi(\xi)}{q(\xi) r(\xi)} \sin \phi_{i} \\
& b_{4,3 i-1}=-\left[c(\xi) h_{s i}^{m}-\frac{q_{i} c_{i}}{q(\xi)} \frac{d h_{B i}^{m}}{d \xi_{3}}\right] \frac{\cos \phi(\xi)}{r(\xi)} \sin \phi_{i} \\
& -\frac{d h_{n i}^{m}}{d \xi} \quad \frac{\cos \phi(\xi)}{q(\xi) r(\xi)} \cos \phi_{i} \text {, and }
\end{aligned}
$$

24 
$b_{4,3 i}=\frac{q_{i}}{q(\xi)} \frac{d h_{B i}^{m}}{d \xi_{3}} \quad \frac{\cos \phi\left(\xi_{3}\right)}{r\left(\xi_{3}\right)}$

where $i=1, \ldots m$.

AJPENDIX B

B MATRIX FOR IISPLACIMMENTS AND MERIDIONAL MOMENT

$b_{1,3 i-2}=\frac{1}{q(\xi)} \frac{d h_{s i}^{m}}{d \xi} \cos \phi_{i}+c(\xi) h_{s i}^{m} \sin \phi_{i}$,

$b_{1,3 i-1}=\frac{-1}{q(\xi)} \frac{d h_{s i}^{m}}{d \xi} \sin \phi_{i}+c(\xi) h_{s i}^{m} \cos \phi_{i}$,

$b_{1,3 i}=0$;

$b_{2,3 i-2}=\frac{\cos \phi(\xi) h_{s i}^{m} \cos \phi_{i}+\sin \phi(\xi) h_{s i}^{m} \sin \phi_{i}}{r(\xi)}-\frac{\text { and }}{2}$

$b_{2,3 i-1}=\frac{\cos \phi(\xi) h_{s i}^{m} \sin \phi_{i}+\sin \phi(\xi) h_{s i}^{m} \cos \phi_{i}}{r(\xi)}$, 
or, using the simplifying assumption $u_{r}(\xi)=\sum_{i=l}^{m} h_{s i}^{m} u_{r i}$,

$b_{2,3 i-2}=\frac{h_{s i}^{m}}{r(\xi)}$.

$b_{2}, 3 i-1=0$,

$b_{2}, 3 i=0 ;$

$b_{3}, 3 i-2=0$,

$b_{3,3 i-1}=0$,

$b_{3,3 i}=h_{s i}^{m}$;

$\mathbf{b}_{4,3 i-2}=\frac{\cos \phi(\xi)}{r(\xi)}\left\{c(\xi) h_{s i}^{m} \cos \phi_{i}-\frac{1}{q(\xi)} \frac{\mathrm{dh}_{\mathrm{si}}^{i}}{\mathrm{~d} \xi} \sin \phi_{i}\right\}$,

$b_{4,3 i-1}=\frac{\cos \phi(\xi)}{r(\xi)}\left\{-c(\xi) h_{s i}^{m} \sin \phi_{i}-\frac{1}{q(\xi)} \frac{d h_{s i}^{m}}{d \xi} \cos \phi_{i}\right\}$,

$b_{4,3 i}=0$; 


$$
\begin{aligned}
& b_{5}, 3 i-2=0 \text {, } \\
& b_{5}, 3 i-1=0 \text {, } \\
& b_{5,3 i}=\frac{1}{q(\xi)} \frac{d h^{m} s i}{d \xi} \text {; } \\
& { }^{b}{ }_{6}, 3 i-2=c(\xi) h_{s i}^{m} \cos \phi_{i}-\frac{1}{q(\xi)} \frac{d h_{s i}^{m}}{d \xi_{2}} \sin \phi_{i} \text {, } \\
& b_{0,3 i-1}=-c\left(\xi_{1}\right) h_{s i}^{m} \sin \phi_{i}-\frac{1}{q(\xi)} \frac{d h_{s i}^{m}}{d \xi} \cos \phi_{i} \text {, and } \\
& b_{6,3 i}=0 \\
& \text { where } i=1 . m \text {. } \\
& \text { APPENDIX } \mathrm{C} \\
& \text { MIXED VARIATIONAL PRINCIPAL }
\end{aligned}
$$

The following variational principle, originally formulated by Hermann, Taylor, and Green in Ref. 5, is an expression for the first two equilibrium equations and the meridional moment constitutive relation. 


$$
\begin{aligned}
\delta I\left(u_{s}, u_{n}, M_{s}\right) & \left.=\delta U \mu_{s}, u_{n}, M_{s}\right)-\int r\left\{s \delta u_{s}+p \delta u_{n}\right\} d s \\
& -r\left[\bar{N}_{s} \delta u_{s}+\bar{Q} \delta u_{n}+\bar{B} \delta M_{s}\right]_{B}=0
\end{aligned}
$$

where

$$
\begin{aligned}
& \delta U\left(u_{s}, u_{n}, M_{s}\right)=\int\left\{r N_{s}\left(\frac{d\left(\delta u_{s}\right)}{d s}+\frac{\delta u_{n}}{R}\right)+N_{\theta} \delta u_{r}\right. \\
& -r\left[\frac{M_{s}}{D_{s}}+\left(1-\frac{D_{s \theta}}{D_{s}}\right) k_{\theta}\right] \delta M_{s}-r \beta \frac{d\left(\delta M_{s}\right)}{d s} \\
& \left.-r \quad Q\left(M_{s}, K_{\theta}\right)\left[\frac{\delta u_{s}}{R}-\frac{d\left(\delta u_{n}\right)}{d s}\right]\right\} \text { and } \\
& Q\left(M_{s}, k_{\theta}\right)=\frac{d_{s}}{d s}+\left[1-\frac{D_{s} \theta}{D_{s}}\right] \frac{\cos \phi}{r} M_{s}-\left[D_{\theta}-\frac{D_{s \theta}^{2}}{D_{s}}\right] \kappa_{\theta} \frac{\cos \phi}{r} \cdot(C-3)
\end{aligned}
$$

Equation ( $\mathrm{C}-3)$ is obtained from the moment and shear equilibrium equation and the $M_{\theta}$ constitutive relation. 
since $\delta u_{r}=\cos \psi \delta u_{s}+\sin \phi \delta u_{n}$ and

$$
\int r f \frac{d g}{d s} d s=[r f g]_{B}-\int g \frac{d(r f)}{d s} d s,
$$

$\therefore\left(u_{s}, u_{n}, M_{s}\right)$ can be written as

$$
\begin{aligned}
\delta \mathrm{r}\left(u_{s}, u_{n}, M_{s}\right) & =\int\left\{\left[-\frac{d\left(r N_{s}\right)}{d s}+N_{\theta} \cos \phi-\frac{r Q}{R}-r s\right] \delta u_{s}\right. \\
& +\left[N_{\theta} \sin \phi-\frac{d(r Q)}{d s}+r \frac{N_{s}}{R}-r p\right] \delta u_{n} \\
& \left.+\left[r\left(-\frac{M_{s}}{D_{s}}+\frac{D_{s \theta}}{D_{s}} k_{\theta}+k_{s}\right)\right] \delta M_{s}\right\} d s \\
& +r\left[\left(N_{s}-\bar{N}_{s}\right) \delta u_{s}+(Q-\bar{Q}) \delta u_{n}+(B-\bar{B}) \delta M_{s}\right]_{B}=0
\end{aligned}
$$

The following relation was used:

$$
\frac{d(r \beta)}{d s}=r k_{\theta}+r k_{s} .
$$


lequation $(C-A)$ is an expression of the meridional and normal equations of equilibrium, the meridional moment ( $M_{s}$ ) constitutive relation, and boundary conditions.

Note that because the relation $\frac{B}{r} \frac{d r}{d s}$ was used for $k_{g}$ in this development [for Eq. (C-5)] and because this is a sinall rotation relation, this section is restricted to small rotation theory.

\section{APPENIIX I)}

POTINTIAI, ENIERGY VARIATIONAL PRINCIPLE:

The theorem of minimum potential energy may be expressed as follows:

$$
\begin{aligned}
& \delta P\left(u_{s}, u_{n}, \beta\right)=\delta U\left(u_{s}, u_{n}, \beta\right)-\int_{L} r\left\{s \delta u_{s}+p \delta u_{n}\right\} d s \\
& -r\left[\bar{N}_{s} \delta u_{s}+\bar{Q} \delta u_{n}+\bar{M}_{s} \delta \beta\right]_{B}=0
\end{aligned}
$$

where

$$
\begin{aligned}
& \delta J\left(u_{s}, u_{n}, \beta\right)=\int_{L}\left\{N_{s}\left(\frac{d\left(\delta u_{s}\right)}{d s}+\frac{\delta u_{n}}{R}\right)+N_{\theta} \frac{\delta u_{r}}{r}+M_{s} \frac{d(\delta \beta)}{d s}\right. \\
& \left.+M_{\theta} \frac{c-j \phi}{r} \delta \beta+Q\left(\frac{d\left(\delta u_{n}\right)}{d s}+\delta \beta-\frac{\delta u_{s}}{R}\right)\right\} d s .
\end{aligned}
$$

That Eq. (D-1) is an expression of equilibrium can be shown as follows:

$\delta u_{r}=\cos \phi \delta u_{s}+\sin \phi \delta u_{n}$, and by integration by parts 


$$
\int_{L} r f \frac{d g}{d s} d s=\left[\begin{array}{lll}
r & f
\end{array}\right]_{B}-\int_{L} \frac{d(r f)}{d s} d s
$$

Making these substitutions into the strain energy expression

$$
\begin{aligned}
& \delta u\left(u_{s}, u_{n}, B\right)(\text { E:q. (I)-2)), } \\
& \delta u\left(u_{s}, u_{n}, \beta\right)=\int\left[-\frac{d\left(r N_{s}\right)}{d s}+N_{\theta} \cos \phi-Q \frac{r}{R}\right] \delta u_{s} \\
& +\left[\frac{r N_{s}}{R}+N_{\theta} \sin \phi-\frac{d(r}{d s}\right) \delta u_{n} \\
& \left.+\left[-\frac{d\left(r M_{s}\right)}{d s}+M_{\theta} \cos \phi+r Q\right] \delta \beta\right\} d s \\
& +r\left[N_{s} \delta u_{s}+Q \delta u_{n}+M_{s} \delta \beta\right]_{B}
\end{aligned}
$$

Substituting Eq. (D-3) into Eq. (D-1) 


$$
\begin{aligned}
\delta P\left(u_{s}, u_{n}, B\right) & =\int\left\{-\frac{d\left(r N_{s}\right)}{d s}+N_{\theta} \cos \phi-\frac{Q r}{R}-r s\right] \delta u_{s} \\
& +\left[\frac{r N_{s}}{R}+N_{\theta} \sin \phi-\frac{d(r Q)}{d s}-r p\right] \delta u_{n} \\
& \left.\left.+-\frac{d\left(r M_{s}\right)}{d s}+M_{\theta} \cos \phi+r Q\right] \delta \beta\right\} d s \\
& +r\left[\left(N_{s}-\bar{N}_{s}\right) \delta u_{s}+(Q-\bar{Q}) \delta u_{n}\right. \\
& \left.+\left(M_{s}-\bar{M}_{s}\right) \delta \beta\right] B
\end{aligned}
$$

Equation (D-4) is an expression for equilibrium and boundary conditions. 


\section{APPENDIX E}

\section{SHAPE FUNCTIONS FOR SHELL ELEMENTS}

Figure E-1 shows the normalized coordinate $\xi$. The $\xi$ designates nodal point 1 when $\xi=1$, nodal point 2 when $\xi_{2}=-1$, and nodal point 3 when $\xi=0$.

For two nodal point elements the mid-side nodal point (nodal point 3 in Fig. E-1) is deleted. Thus for assumed displacement functions let

$$
u_{s}=a+b \xi \quad \text { and } \quad u_{n}=c+d \xi_{s}+e \xi^{2}+f \xi^{3} \text {. }
$$

$a, b, c, d, e$, and $f$ can be solved for from the following conditions,

$$
\begin{array}{ll}
\xi=1 & u_{s}=u_{s 1}, \\
\xi=-1 & u_{s}=u_{s 2}, \\
\xi=1 & u_{n}=u_{n 1}, \\
\xi=-1 & u_{n}=u_{n 2}, \\
\xi=1 & \frac{d u_{n}}{d \xi}=\psi_{1}, \\
\xi=-1 & \frac{d u_{n}}{d \xi}=\psi_{2} .
\end{array}
$$

Thus $u_{s}=\sum_{i=1}^{2} h_{s i}^{2} u_{s i}$ and $u_{n}=\sum_{i=1}^{2} h_{n i}^{2} u_{n i}+h_{B_{i}}^{2} \psi_{i}$

where the shape functions $h_{s i}^{2}, h_{n i}^{2}$, and $h_{B}^{2}$ are

$$
\begin{array}{ll}
h_{s 1}^{2}=\frac{1}{2}(1+\xi), & h_{n 1}^{2}=\frac{1}{4}\left(2+3 \xi-\xi^{2}\right), \\
h_{s 2}^{2}=\frac{1}{2}(1-\xi), & h_{B 1}^{2}=\frac{1}{4}\left(-1-\xi+\xi^{2}+\xi^{3}\right)
\end{array}
$$




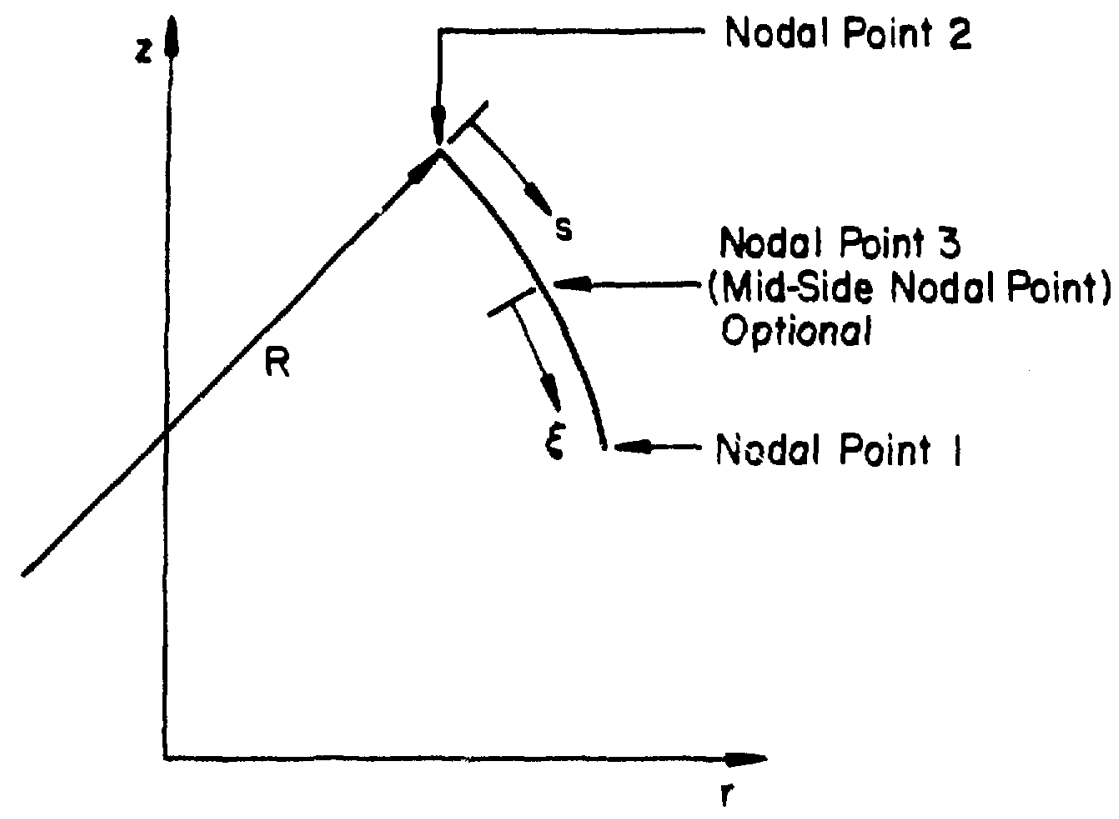

Fig. li-l. Normalized coordinate (f) for symmetrically loaded shell of revolution model.

and the superscript designates the number of nodal points in the element.

For three nodal point clements the assumed displacement functions are

$$
u_{s}=a+b \xi+c \xi^{2} \quad \text { and } \quad u_{n}=d+c \xi_{s}+f \xi^{2}+g \xi^{3}+h \xi_{s}^{4}+i \xi^{5} \text {. }
$$

The constants $a, b, c, d, c, f, g, h$, and $i$ can be solved for from the following conditions :

$$
\begin{array}{ll}
\xi=1 & u_{s}=u_{s 1}, \\
\xi=-1 & u_{s}=u_{s 2}, \\
\xi=0 & u_{s}=u_{s 3}, \\
\xi=1 & u_{n}=u_{n 1}, \\
\xi=-1 & u_{n}=u_{n 2}, \\
\xi=0 & u_{n}=u_{n 3},
\end{array}
$$




$$
\begin{array}{ll}
\xi=1 & \frac{d u_{n}}{d \xi}=\psi_{1}, \\
\xi=-1 & \frac{d u_{n}}{d \xi} \cdot \psi_{2} . \\
\xi=0 & \frac{d u_{n}}{d \xi}=\psi_{3} .
\end{array}
$$

Thus $u_{s}=\sum_{i=1}^{3} h_{s i}^{3} u_{s i}$ and $u_{n}=\sum_{i=1}^{3}\left(h_{n i}^{3} u_{n i}+h_{\beta_{j}}^{3} \psi_{i}\right)$,

where the shape functions $h_{s i}^{3}, h_{n i}^{3}$, and $h_{f i}^{3}$ are

$$
\begin{aligned}
h_{s 1}^{3} & =\frac{1}{2} \xi\left(1+\xi_{3}\right), \quad h_{n 1}^{3}=\frac{1}{4} \xi^{2}\left(4+5 \xi-2 \xi^{2}-3 \xi^{3}\right), \\
h_{s 2}^{3}=-\frac{1}{2} \xi(1-\xi), & h_{n 2}^{3}=\frac{1}{4} \xi^{2}\left(4-5 \xi-2 \xi^{2}+3 \xi^{3}\right), \\
h_{s 3}^{3}=1-\xi^{2}, & h_{n 3}^{3}=\left(1-\xi^{2}\right)^{2}, \\
h_{B 1}^{3} & =1 / 4 \xi^{2}\left(-1-\xi+\xi^{2}+\xi^{3}\right), \\
h_{B 2}^{3} & =1 / 4 \xi^{2}\left(1-\xi-\xi^{2}+\xi^{3}\right), \\
h_{B 3}^{3} & =\xi\left(1-\xi^{2}\right)^{2},
\end{aligned}
$$

and the superscript designates the number of nodal points in element.

At this point the variable $\psi_{i}$ should be replaced with the rotation $B_{i}$. The definition of rotation (for small rotations) is

$$
B(\xi)=\frac{u_{s}(\xi)}{R(\xi)} \cdot \frac{d u_{n}(\xi)}{d s} .
$$


Let $q(\xi) d \xi=d s$ and $c(\xi)=\frac{1}{R(\xi)}$; then

$$
B(\xi)=c(\xi) \quad u_{s}(\xi)-\frac{1}{q(\xi)} \frac{d u_{n}(\xi)}{d \xi} .
$$

Since

$$
\begin{aligned}
& u_{s}(\xi)=\sum_{i=1}^{m} h_{s i}^{n} u_{s i} \text { and } u_{n}(\xi)=\sum_{i=1}^{m}\left(h_{n i}^{m} u_{n i}+h_{B i}^{m} \psi_{i}\right), \\
& B(\xi)=c(\xi) \sum_{i=1}^{m} h_{s i}^{m} u_{s i}-\frac{1}{q(\xi)} \sum_{i=1}^{m}\left(\frac{d n_{n i}^{m}}{d \xi} u_{n i}+\frac{d h_{B i}^{m}}{d \xi} \psi_{i}\right) .
\end{aligned}
$$

If $\xi_{k}$ is the value of $\xi$ at a nodal point then $h_{s i}^{m}\left(\xi_{k}\right)=\delta_{i k}$,

$\left.\frac{d h_{n i}^{m}}{d \xi}\right|_{\xi=\xi_{k}}=0$, and $\left.\frac{d h_{B i}^{m}}{d \xi}\right|_{\xi=\xi_{k}}=\delta_{i k}$. Let $\beta\left(\xi_{k}\right)=\beta_{k}$,

$$
c\left(\xi_{k}\right)=c_{k} \text {, and } q\left(\xi_{k}\right)=q_{k} \text {. }
$$

Then

$$
\beta_{k}=c_{k} u_{s k}-\frac{\psi_{k}}{q_{k}} \quad \text { and } \quad \psi_{k}=q_{k}\left(c_{k} u_{s k}-\beta_{k}\right) .
$$

The displacements and rotation may be approximated as

$$
u_{s}(\xi)=\sum_{i=1}^{m} h_{s i}^{m} u_{s i}
$$




$$
\begin{aligned}
& u_{n}(\xi)=\sum_{i=1}^{m}\left\{h_{B i}^{m} q_{i} c_{i} u_{s i}+h_{n i}^{m} u_{n i}-h_{B i}^{m} q_{i} \beta_{i}\right\}, \\
& \beta(\xi)=\sum_{i=1}^{m}\left\{\left[c(\xi) h_{s i}^{m}-\frac{1}{q(\xi)} q_{i} c_{i} \frac{d h_{\beta_{j}}^{m}}{d \xi}\right] u_{s i}\right. \\
& \left.-\frac{1}{q(\xi)} \frac{d h_{n i}^{m}}{d \xi} u_{n i}+\frac{q_{i}}{q(\xi)} \frac{d h_{\beta i}^{m}}{d \xi} \beta_{i}\right\} \text {. } \\
& \text { APPENDIX F } \\
& \text { EQUILIBRIUM EQUATIONS }
\end{aligned}
$$

There are three equilibrium equations for a symmetrically loaded shell of revolution as shown in Figs. F-1 - F-3. These are equilibrium along the meridional coordinate $s$, the normal coordinate $n$, and rotational equilibrium.

Equilibrium along the meridional coordinate

$$
\begin{aligned}
& -N_{s} \cos (\Delta \phi / 2) r \Delta \theta+\left(N_{s}+\Delta N_{s}\right) \cos (\Delta \phi / 2)(r+\Delta r) \Delta \theta \\
& -N_{\theta} \Delta \theta \Delta s \cos \phi+s(r+\Delta r / 2) \Delta \theta \Delta s+(Q+\Delta Q) \sin (\Delta \phi / 2)(r+\Delta r) \Delta \theta \\
& +Q \sin (\Delta \phi / 2) r \Delta \theta=0 .
\end{aligned}
$$




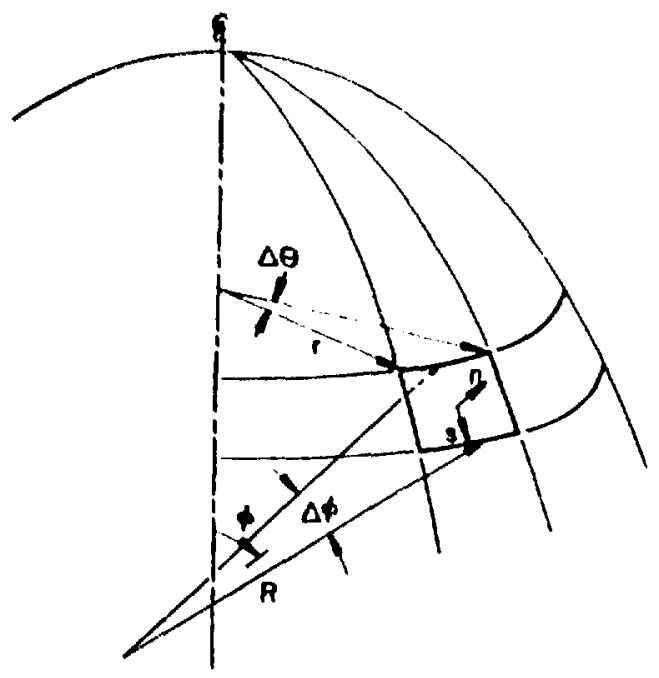

Fig. $F-1$. Cometry for symmetricilly loaded shell of revolution.

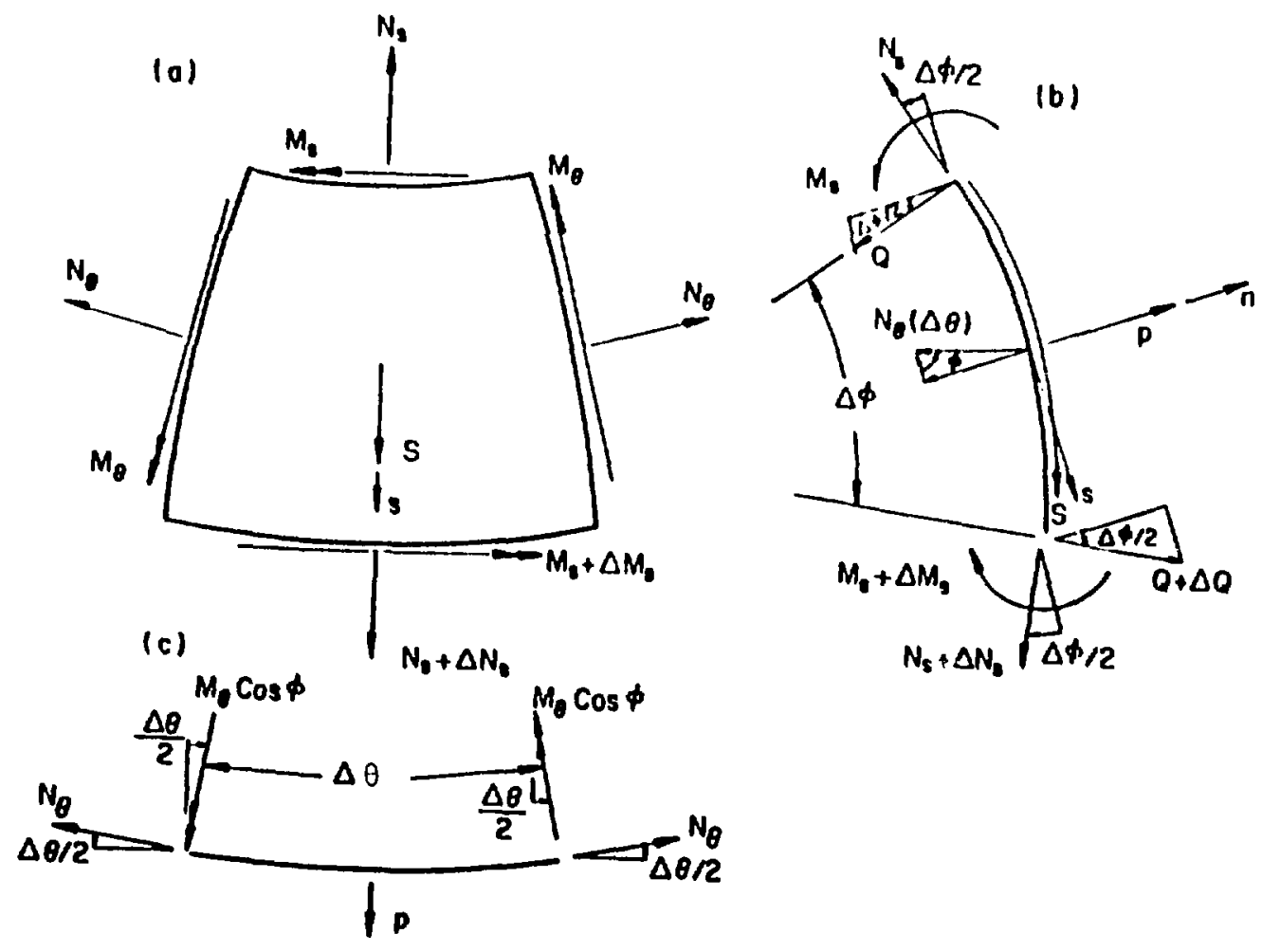

Fig. F-3. Geometry, stress resultants, bending moments, and loads for a symmetrically loaded shell of revolution. 
As $\Lambda \phi$ approaches zero, $\sin (\Delta \phi / 2)$ approaches $\Delta \phi / 2$ and $\cos (\Delta \phi / 2)$ approaches the value 1. Also, since $k \Delta \phi=A s$ and $\Delta \phi=\frac{\Delta s}{R}$,

$$
\begin{aligned}
& -N_{s} r \Delta \theta+N_{s} r \Delta \theta+\Delta N_{s} r \Delta \theta+N_{s} \Delta r \Delta \theta+\Delta N_{s} \Delta r \Delta \theta \\
& -N_{0} \Delta \theta \Delta s \cos \phi+s r \Delta \theta \Delta s+s \frac{\Delta r}{2} \Delta \theta \Delta s+Q \frac{\Delta s}{2 R} r \Delta \theta+Q \frac{\Delta s}{2 R} \Delta r \Delta \theta \\
& +\Delta Q \frac{\Delta s}{2 R} r \Delta \theta+\Delta Q \frac{\Delta s}{2 R} \Delta r \Delta \theta+Q \frac{\Delta s}{2 R} r \Delta \theta=0 .
\end{aligned}
$$

Simplifying and dividing this equation by $\Lambda s$,

$$
\begin{aligned}
& \frac{\left(\Delta N_{s} r+N_{s} \Lambda r\right)}{\Delta s}-N_{0} \cos \phi+Q \frac{r}{R}+S r+\frac{\Delta N_{s} \Delta r}{\Delta s}+\frac{s}{2} \Delta r+\frac{Q}{2 R} \Delta r+\frac{\Delta Q r}{2 R} \\
& +\frac{\Delta Q \Delta r}{2 R}=0 .
\end{aligned}
$$

As $\Delta s$ approaches zero and $\Delta \theta$ approaches zero,

$$
\frac{\Delta N_{s} r+N_{s} \Delta r}{\Delta s} \rightarrow \frac{d\left(r N_{s}\right)}{d s}, \frac{\Delta N_{s} \Delta r}{\Delta s} \rightarrow \frac{d N_{s}}{d s} \Delta r \rightarrow 0
$$$$
\Delta Q \rightarrow 0 \text { and } \Delta r \rightarrow 0 \text {. Thus }
$$$$
\frac{\mathrm{d}\left(\mathrm{r} \mathrm{N}_{\mathrm{s}}\right)}{\mathrm{ds}}-\mathrm{N}_{\theta} \cos \phi+Q \frac{\mathrm{r}}{\mathrm{R}}+\mathrm{s} \mathrm{r}=\mathrm{C}
$$ 
Equilibrium along the normal coordinate

$$
\begin{aligned}
& (Q+\Delta Q) \cos (\Lambda \phi / 2)(r+\Delta r) \Delta \theta-Q \cos (\Delta \phi / 2) r \Delta \theta-N_{0} \Delta \theta \sin \phi \Delta s \\
& -\left(N_{s}+\Delta N_{s}\right) \sin (\Delta \phi / 2)(r+\Delta r) \Delta \theta-N_{s} \sin (\Delta \phi / 2) r \Delta \theta \\
& +p \Delta s(r+\Delta r / 2) \Delta \theta=0 .
\end{aligned}
$$

Simplifying as before,

$$
\begin{aligned}
\frac{Q \Delta r+r \Delta Q}{\Delta s}- & N_{\theta} \sin \phi-\frac{N_{s} r}{R}+p r+\frac{\Delta Q}{\Delta s} \Delta r-\frac{N_{S} \Delta r}{2 R}-\frac{N_{s}}{2 R}(r+\Delta r) \\
& +\frac{p}{2} \Delta r=0 .
\end{aligned}
$$

In the limit

$$
\frac{\mathrm{d}(\mathrm{r} 0)}{\mathrm{ds}}-\mathrm{N}_{\theta} \sin \phi-\frac{\mathrm{N}_{\mathrm{s}} \mathrm{r}}{\mathrm{R}}+\mathrm{pr}=0 .
$$

Rotational equilibriun

$$
\begin{aligned}
& \left(M_{s}+\Delta M_{s}\right)(r+\Delta r) \Delta \theta-M_{s} r \Delta \theta-M_{\theta} \cos \phi \Delta s \Delta \theta \\
& -(Q+\Delta Q)(r+\Delta r) \Delta \theta \frac{\Delta s}{2}-Q r \Delta \theta \frac{\Delta s}{2}=0 .
\end{aligned}
$$

\section{Simplifying as before,}




$$
\frac{M_{s} \Delta_{r}+M_{s} r}{\Delta s}-M_{\theta} \cos \phi-Q r+\frac{\Delta M_{s} \Delta r}{\Delta s}-(Q+\Delta Q) \frac{\Delta r}{2}-\Delta Q \frac{r}{2}=0 .
$$

In the linit

$$
\frac{d\left(r M_{s}\right)}{d s}-M_{0} \cos \phi-Q r=0
$$

These equilibrium equations written in this form apply for a circular plate, cylinder, and any other general shell of revolution that is symetrically loaded and has symmetrical boundary conditions. See Refs. 7 and 8 for additional discussion concerning these equilibrium equations.

When $r, z, \phi, s$, and $R$ refer to the deformed configuration, the equilibrium ligs, $(F-1)-(F-3)$ are valid for nonlinear geometric shells.

\author{
APPENDIX $G$ \\ GEOMETRIC QUANTITIES REQUIRED FOR STIFFNESS \\ AND FORCE MODIFICATION CALCULATIONS
}

Two approaches are described for determining the geometric quantities used for the shell stiffness and force modification calculations. (See pgs. 16-22 of text). These are 


\section{Approach 1}

Item 2: Input the coordinates $r$ and $z$ at each nodal point and assunc

$$
r\left(E_{3}\right)=\sum_{i=1}^{m} h_{s i}^{\mathrm{rn}} r_{i} \quad \text { and }
$$

$$
z(\xi)=\sum_{i=1}^{m} h_{s i}^{m}{ }_{i},
$$

then

$$
\frac{d r(\xi)}{d \xi}=\sum_{i=1}^{m} \frac{d h_{s i}^{m}}{d \xi} r_{i} \quad \text { and }
$$

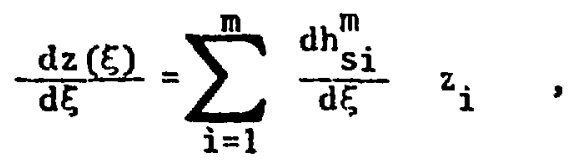

and

$$
\frac{d^{2} r(\xi)}{d \xi^{2}}=\sum_{i=1}^{m} \frac{d^{2} h_{s i}^{m}}{d \xi^{2}} r_{i} \quad \text { and }
$$

$$
\frac{d^{2} z(\xi)}{d \xi^{2}}=\sum_{i=1}^{m} \frac{d^{2} h_{s i}^{m}}{d \xi^{2}} z_{i}
$$


ltem 3: The normalized meridional length $q(\varepsilon)$ is defined as follows:

$$
q\left(\xi_{3}\right)=\frac{d s}{d \xi_{s}}=\sqrt{\left(\frac{d r\left(\xi_{2}\right)}{d \xi_{3}}\right)^{2}+\left(\frac{d z\left(\xi_{)}\right.}{d \xi_{3}}\right)^{2}}
$$

and

$$
\frac{d}{d \xi}\left(\frac{1}{q(\xi)}\right)=\frac{-\frac{d^{2} z(\xi)}{d \xi^{2}} \frac{d z(\xi)}{d \xi}-\frac{d^{2} r(\xi)}{d \xi^{2}} \frac{d r(\xi)}{d \xi}}{(q(\xi))^{3}} .
$$

Item 4: The azimuthal angle $\phi(\xi)$ is determined as

$$
\cos \phi(\xi)=\frac{d r}{d s}=\frac{1}{q(\xi)} \quad \frac{d r(\xi)}{d \xi} \quad \text { and }
$$

$$
\sin \phi(\xi)=-\frac{d z}{d s}=\frac{1}{q(\xi)} \quad \frac{d z(\xi)}{d \xi} .
$$

Item 5: The curvature $c(\xi) \equiv \frac{1}{R(\xi)}=\frac{\frac{d^{2} z(\xi)}{d \xi^{2}} \frac{d r(\xi)}{d \xi}+\frac{d^{2} r(\xi)}{d \xi^{2}} \frac{d z(\xi)}{d \xi}}{(q(\xi))^{3}}$.

For the derivative of the curvature assume

$$
c(\xi)=\sum_{i=1}^{m} h_{s i}^{m} c_{i}
$$


where $c_{i}=c\left(\xi_{i}\right)$,

then $\quad \frac{\mathrm{dc}\left(\xi_{2}\right)}{\mathrm{d} \xi_{2}}=\sum_{i=1}^{m} \frac{\mathrm{uh}_{\mathrm{si}}^{\mathrm{m}}}{\mathrm{d} \xi_{2}} c_{i}$.

\section{Approach 2}

Item 2: Same as approach 1

Item 4: Input the azimuthal angle $\phi$ at each nodal point $\left(\phi_{i}\right)$, then assume

$$
\phi(\xi)=\sum_{i=1}^{m} h_{s i}^{m} \phi_{i}
$$

then

$$
\frac{d \phi(\xi)}{d \xi}=\sum_{i=1}^{m} \frac{d h_{s i}^{m}}{d \xi_{i}} \phi_{i} \quad \text { and } \quad \frac{d^{2} \phi(\xi)}{d \xi^{2}}=\sum_{i=1}^{m} \frac{d^{2} h_{s i}^{m}}{d \xi^{2}} \phi_{i} \text {. }
$$

Item 5: Input the curvature $c$ at each nodal point $\left(c_{i}\right)$ then assune

$$
c(\xi)=\sum_{i=1}^{m} h_{s i}^{m} c_{i}
$$

then

$$
\frac{\mathrm{dc}(\xi)}{\mathrm{d} \xi}=\sum_{i=1}^{\mathrm{m}} \frac{\mathrm{dh} \mathbf{s i}}{\mathrm{d} \xi} \quad c_{i}
$$


Item 3: The nommalized meridional length $q(\xi)$ is defined as

$$
q(\xi) d \xi \equiv R(\xi) d \phi
$$

thus

$$
\begin{gathered}
q(\xi)=\frac{1}{c(\xi)} \frac{d \phi(\xi)}{d \xi} \text {, and } \\
\frac{d}{d \xi}\left(\frac{1}{q(\xi)}\right)=\frac{\frac{d c(\xi)}{d \xi} \quad \frac{d \phi(\xi)}{d \xi}-e(\xi) \frac{d^{2} \phi(\xi)}{d \xi^{2}}}{\left(\frac{d \varphi(\xi)}{d \xi}\right)^{2}} .
\end{gathered}
$$

For plates and cylinders $c(\xi)=0 . \phi(\xi)=0$ for a plate and $\phi(\xi)=\pi / 2$ for a cylinder. In these cases $q(\xi)$ is input for each nodal point of the element $\left(q_{i}\right)$, and then assume

$$
\frac{1}{q(\xi)}=\sum_{i=1}^{m} h_{s i}^{m} \frac{1}{q_{i}} \quad \text { then } \quad \frac{d}{d \xi}\left(-\frac{1}{q(\xi)}\right)=\sum_{i=1}^{m} \frac{d h_{s i}^{m}}{d \xi} \frac{1}{q_{i}}
$$




\section{APPENDIX $\|$}

\section{GEOMETRIC NONLINEAR SHELL QUANTITIES}

One of the basic assumptions used in geometric linear shell theory is that the initial and final geometry are essentially the same. Thus, for geometric nonlinear shell theory, the geometric and shell quantities must be identified as to whether they refer to deformed or undeformed geometry. All quantities in this report will refer to deformed geometry unless they are subscripted with a "o" in which case they will refer to undeformed geometry.

Therefore, the geometric quantities used to describe a shell are

- the coordinates $r, z$ and $r_{0}, z_{0}$;

- the angle from the axis of revolution $\phi$ and $\phi_{0}$;

- the meridional coordinate $s$ and $s_{0}$;

- the normalized meridional length increment $q$ and $q_{0}$; and

- the radius of curvature $R$ and $R_{0}$.

The shell quantities are

- the membrane strains $\varepsilon_{s}, \varepsilon_{\theta}$ and $\varepsilon_{s_{0}}, \varepsilon_{\theta_{0}}$;

- the bending strains $k_{s}, k_{\theta}$ and $k_{s_{0}}, k_{\theta}$;

- the stress resultants, $\mathrm{N}_{\mathrm{s}}, \mathrm{N}_{\theta}$ and $\mathrm{N}_{\mathrm{s}_{0}}, \mathrm{~N}_{\theta}$; and

- the bending moments $M_{s}, M_{\theta}$ and $M_{s_{0}}, M_{\theta}$.

Let the membrane and bending strains be defined as follows (see Ref. 9):

- $\varepsilon_{s}=\frac{q-q_{0}}{q}, \quad \varepsilon_{\theta}=\frac{r-r_{0}}{r}$

- $k_{s}=\frac{d\left(\phi-\phi_{0}\right)}{d s}, \quad \kappa_{\theta}=\frac{\sin \phi-\sin \phi_{0}}{r}$ 
and

$$
\begin{array}{rlr}
-r_{s_{0}}=\frac{q-q_{0}}{q_{0}}, & \varepsilon_{0}=\frac{r-r_{0}}{r_{0}} \\
-r_{s_{0}}=\frac{d\left(\phi-\phi_{0}\right)}{d s_{0}}, & r_{0}=\frac{\sin \phi-\sin \phi_{0}}{r_{0}}
\end{array}
$$

(as $r, r_{0}$ approach zero; see Appendix L.)

Some interesting transformations between the deformed and undeformed quantities are:

$$
\begin{array}{ll}
d s=\frac{q}{q_{0}} d s_{0} & d s_{0}=\frac{q_{0}}{q} d s \\
d s=\left(1+\varepsilon_{s_{0}}\right) d s_{0} & d_{s_{0}}=\left(1-\varepsilon_{s}\right) d s \\
q=q_{0}\left(1+\varepsilon_{s_{0}}\right) & q_{0}=q\left(1-\varepsilon_{s}\right) \\
r=r_{0}\left(1+\varepsilon_{\theta}\right) & r_{0}=r\left(1-\varepsilon_{\theta}\right) \\
\varepsilon_{s}=\frac{\varepsilon_{s}}{1+\varepsilon_{s}} & \varepsilon_{s_{0}}=\frac{\varepsilon_{s}}{1-\varepsilon_{s}}
\end{array}
$$




$$
\begin{aligned}
& \varepsilon_{\theta}=\frac{\varepsilon_{0}}{1+\frac{\varepsilon_{0}}{1+\varepsilon_{0}}} \\
& k_{s}=\frac{k_{s_{0}}}{1+\varepsilon_{s_{0}}} \\
& k_{\theta}=\frac{\kappa_{\theta}}{1+\varepsilon_{\theta}}
\end{aligned}
$$

Differential relations

$$
d s=R \quad d \phi=q \quad d \varepsilon_{,},
$$

$$
\varepsilon_{\theta}=\frac{\varepsilon_{\theta}}{1-\varepsilon_{\theta}}
$$

$$
k_{s_{0}}=\frac{k_{s}}{1-s_{s}}
$$

$$
\kappa_{\theta}=\frac{\kappa_{\theta}}{1-\varepsilon_{\theta}} \text {. }
$$

$$
d s_{0}=R_{0} d \phi_{0}=q_{0} d \xi_{2}
$$

APPENDIX I

INCREMENTING AND LINEARIZING OF $\varepsilon_{s}, \varepsilon_{\theta}, K_{s}$, AND $\kappa_{\theta}$ FOR NONLINEAR SHELLS

Incrementing

$$
\begin{aligned}
\text { Since } \varepsilon_{s} & =\frac{q-q_{0}}{q} \text { (see Appendix } H \text { ), for the } j+1 \text { increment } \\
\varepsilon_{s_{j+1}} & =\frac{q_{j+1}-q_{0}}{q_{j+1}}=\frac{q_{j}}{q_{j+1}}\left\{\frac{q_{j}-q_{0}}{q_{j}}+\frac{q_{j+1}-q_{j}}{q_{j}}\right\} \\
& =\frac{1}{1+\Delta \varepsilon_{s_{j}}}\left\{\varepsilon_{s_{j}}+\Delta \varepsilon_{s_{j}}\right\}, \Delta \varepsilon_{s_{j}}=\frac{q_{j+1}-q_{j}}{q_{j}} .
\end{aligned}
$$

48 
Similarly, since $\varepsilon_{\theta}=\frac{r-r_{0}}{r}$, for the $j+1$ increment,

$$
\begin{aligned}
& \varepsilon_{\theta_{j+1}}=\frac{r_{j+1}-r_{0}}{r_{j+1}}=\frac{r_{j}}{r_{j+1}}\left\{\frac{r_{j}-r_{0}}{r_{j}}+\frac{r_{j+1}-r_{j}}{r_{j}}\right\} \\
& =\frac{1}{1+\Delta \varepsilon_{\theta}}\left\{\varepsilon_{\theta_{j}}+\Delta \varepsilon_{\theta_{j}}\right\}, \quad \Delta \varepsilon_{\theta_{j}}=\frac{r_{j+1}-r_{j}}{r_{j}} . \\
& \text { Also, since } k_{s}=\frac{d\left(\phi_{j}-\phi_{0}\right)}{d s}=\frac{1}{q} \frac{d\left(\phi-\phi_{0}\right)}{d \xi} \text {. } \\
& k_{s_{j+1}}=\frac{q_{j}}{q_{j+1}}\left\{\frac{1}{q_{j}} \frac{d\left(\phi_{j}-\phi_{0}\right)}{d_{\xi}}+\frac{1}{q_{j}} \frac{d\left(\phi_{j+1}-\phi_{j}\right)}{d \xi}\right\} \\
& =\frac{1}{1+\Delta \varepsilon s_{j}}\left\{k_{s_{j}}+\Delta k_{s_{j}}\right\}, \quad \Delta k_{s_{j}}=\frac{1}{q_{j}} \frac{d\left(\phi_{j+1}-\phi_{j}\right)}{d \xi} . \\
& \text { Again, since } \kappa_{\theta}=\frac{\sin \phi-\sin \phi_{0}}{r} \text {, }
\end{aligned}
$$




$$
\begin{aligned}
k_{\theta_{j+1}} & =\frac{r_{j}}{r_{j+1}}\left\{\frac{\sin \phi_{j} \cos \left(\phi_{j+1}-\phi_{j}\right)-\sin \phi_{0}}{r_{j}}\right. \\
& \left.+\frac{\sin \left(\phi_{j+1}-\phi_{j}\right) \cos \phi_{j}}{r_{j}}\right\} \\
& =\frac{1}{1+\Delta \varepsilon_{\theta}}\left\{\frac{\sin \phi_{j} \cos \left(\phi_{j+1}-\phi_{j}\right)-\sin \phi_{0}}{r_{j}}\right. \\
& +\frac{\sin \left(\phi_{j+1}-\phi_{j}\right) \cos \phi_{j}}{r_{j}} .
\end{aligned}
$$

$$
\text { Let } \begin{aligned}
\Delta u_{r_{j}} \equiv r_{j+1}-r_{j}, \Delta u_{z_{j}} \equiv z_{j+1}-z_{j}, \\
\Delta B_{j} \equiv \phi_{j+1}-\phi_{j} \text {. Then } \\
\Delta u_{n_{j}}=\sin \phi \Delta u_{r_{j}}+\cos \phi \Delta u_{z_{j}} \text { and } \\
\Delta u_{s_{j}}=\cos \phi \Delta u_{r_{j}}-\sin \phi \Delta u_{z_{j}} .
\end{aligned}
$$

50 
Linearizing

The linearizing assumptions are:

- $\Delta \varepsilon_{\mathbf{s}_{\mathrm{j}}}$ and $\Delta \varepsilon_{\theta_{\mathrm{j}}}$ are small compared to l,

or $\frac{r_{j}}{r_{j+1}} \cong 1 \quad$ and $\quad \frac{q_{j}}{q_{j+1}} \cong 1$;

- $\Delta \beta_{\mathbf{j}} \equiv \phi_{\mathbf{j}+1}-\phi_{\mathbf{j}}$, is a small angle such that $\cos \left(\Delta \beta_{j}\right) \cong 1$ and

$\sin \left(\Delta \beta_{j}\right) \cong \Delta \beta_{j} ;$ and

- $q_{j+1}=\left(R_{j}+\Delta u_{n_{j}}\right) \frac{d \phi_{j}}{d \xi_{j}}+\frac{d}{d \xi_{s}}\left(\Delta u_{j}\right)$ (see Ref. 9).

Thus

$$
\begin{array}{ll}
\varepsilon_{s_{j+1}} \cong \varepsilon_{s_{j}}+\Delta \varepsilon_{s_{j}}, \text { where } \Delta \varepsilon_{s_{j}}=\frac{\Delta u_{n_{j}}}{R_{j}}+\frac{d\left(\Delta u_{j}\right)}{d s_{j}} ; \\
\varepsilon_{\theta_{j+1}} \cong \varepsilon_{\theta_{j}}+\Delta \varepsilon_{\theta_{j}}, \text { where } \quad \Delta \varepsilon_{\theta_{j}}=\frac{\Delta u r_{j}}{r_{j}} ; \\
k_{s_{j+1}} \cong \kappa_{s_{j}}+\Delta k_{s_{j}} \text {, where } \quad \Delta \kappa_{s_{j}}=\frac{d\left(\Delta \beta_{j}\right)}{d s_{j}} ; \text { and } \\
k_{\theta_{j+1}} \cong \kappa_{\theta_{j}}+\Delta k_{\theta_{j}} \text {, where } & \Delta k_{\theta_{j}}=\frac{\Delta \beta_{j} \cos \phi_{j}}{r_{j}} .
\end{array}
$$

51 
APPENDIX $\mathrm{J}$

INCREMENTAL $N_{s}, N_{\theta}, M_{s}$, AND $M_{\theta}$

Consider the following constitutive relations:

$$
N_{s}=C_{s} \varepsilon_{s}+C_{s \theta} \varepsilon_{\theta}+N_{s}
$$

$$
N_{\theta}=C_{s \theta} \varepsilon_{s}+C_{\theta} \varepsilon_{\theta}+N_{\theta}
$$

$$
\begin{aligned}
& M_{s}=D_{s} K_{s}+D_{s \theta} K_{\theta}+M_{s} \\
& M_{\theta}=D_{s \theta} K_{s}+D_{\theta} K_{\theta}+M_{\theta} .
\end{aligned}
$$

The $j+1$ incremental form of these equations is

$$
\begin{aligned}
N_{s}+1 & =C_{s} \varepsilon_{s_{j+1}}+C_{s \theta} \varepsilon_{\theta j+1}+\left(N_{s_{i}}\right)_{j+1} \\
& =C_{s}\left(\varepsilon_{s_{j}}+\Delta \varepsilon_{s_{j}}\right)+C_{s \theta}\left(\varepsilon_{\theta_{j}}+\Delta \varepsilon_{\theta}\right)+\left(N_{s_{j}}\right)_{j+1} \\
& =N_{s_{j}}+C_{s} \Delta \varepsilon_{s_{j}}+C_{s \theta} \Delta \varepsilon_{\theta_{j}}+\left(N_{s_{j}}\right)_{j+1} \\
& =N_{s}+\left(N_{s}\right)_{j+1}+\Delta N_{s},
\end{aligned}
$$

52 
where $\Delta N_{s_{j}}=C_{s} \Delta \varepsilon_{s_{j}}+C_{s \theta} \Delta \varepsilon_{\theta_{j}}$.

Similarly,

$$
N_{\theta_{j+1}}=N_{\theta_{j}}+\left(N_{\theta_{i}}\right)_{j+1}+\Delta v_{\theta_{j}}
$$

$$
\begin{aligned}
\text { where } \Delta N_{\theta} & =C_{s_{\theta}} \Delta \varepsilon_{s_{j}}+C_{\theta} \Delta \varepsilon_{\theta}, \\
N_{s_{j+1}} & =M_{s_{j}}+\left(M_{s_{i}}\right)_{j+1}+M_{s_{j}},
\end{aligned}
$$

where $\Delta M_{s_{j}}=D_{s} \Delta K_{s_{j}}+n_{s \theta} \Delta K_{\theta_{j}}$.
\[ \begin{array}{c}M_{\theta_{j+1}}=M_{\theta_{j}}+\left(M_{\theta_{i}}\right)_{j+1}+\Delta M_{\theta_{j}},\end{array} \]

where $\Delta M_{\theta_{j}}=D_{s \theta} \Delta K_{s_{j}}+D_{\theta} \Delta K_{\theta_{j}}$.

APPENDIX $K$

NONLINEAR MATERIAL PROPERTIES

Consider the nonlinear material shown in Fig. $K-1$. The stress resultant $N_{s}$ refers to the deformed shell configuration, while the membrane strain $E_{s}$ refers to the undeformed shell configuration. The stress resultants and bending

53 


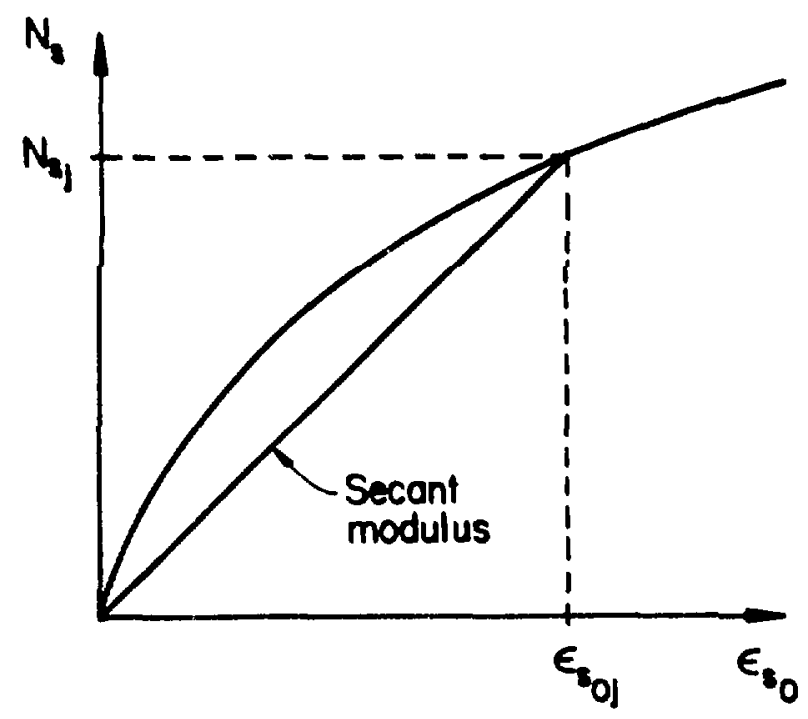

Fig. $k-1$. Nonlinear material $\left(N_{s}\right.$ vs $\left.\varepsilon_{s_{0}}\right)$.

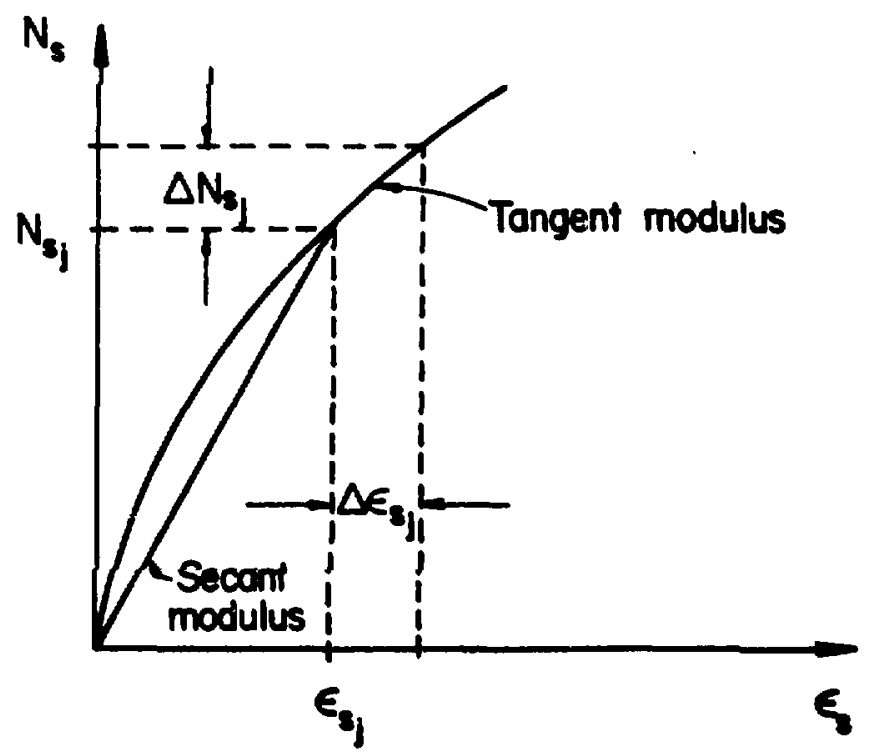

Fig. K-2. Nonlinear naterial ( $N_{s}$ vs $\left.\varepsilon_{s}\right)$ 
moments that refer to the deformed shell configuration and the membrane and hending strains that refer to the undeformed shell configuration are the ones usually associated with experimental work. In this report the membrane and bending strains used are those referring to the deformed shell configuration. Thus, using the transformation shown in Appendix $H$, the nonlinear material properties can be transforned to relate membrane and bending strains to stress resultants and bending monents where all quantities refer to the deformed shell configuration. Using the transformation from $\varepsilon_{S_{0}}$ to $\varepsilon_{s}$, Fig. $K-2$ is generated.

From these two figures it is apparent that $\mathrm{N}_{s_{j}}$ may be obtained using the secant modulus show in either Fig. $K-1$ or $K-2$, while for $\Delta N_{s_{j}}$ and $\Delta \varepsilon_{s_{j}}$ the tangential modulus shown in Fig. $k-2$ must be used. These same conclusions can be made for all material properties. Thus, given the membrane and bending strains in either the deformed or undeformed shell configuration, the deformed shell configuration membrane stresses and bending moments may be obtained using the corresponding secant material properties. lowever, for incremental membrane and hending strains the tangential material properties for the deformed shell configuration quantititos are required.

\section{APPENDIX L $\varepsilon_{\theta}$ AND $K_{\theta}$ FOR CLOSED $(r=0)$ SHELLS}

When a shell of revolution is closed $(r=0)$, special considerations are required to determine the hoop strain $\varepsilon_{\theta}$ and the change of curvature $k_{\theta}$. Since $\frac{\mathrm{dr}}{\mathrm{ds}}=\cos \phi$, the equilibrium Eqs. $(\mathrm{F}-1)$ and $(\mathrm{F}-3)$ become

$$
\begin{aligned}
& N_{s}=N_{\theta} \text { and } \\
& M_{s}=M_{\theta}
\end{aligned}
$$

as $r$ approaches zero. Thus for the constitutive relation of 


$$
\begin{aligned}
& N_{s}=C_{s} \varepsilon_{s}+C_{s \theta} \varepsilon_{\theta}+N_{s i} \\
& N_{\theta}=C_{s \theta} \varepsilon_{s}+C_{\theta} \varepsilon_{\theta}+N_{\theta i} \\
& M_{s}=D_{s} K_{s}+D_{s \theta} K_{\theta}+M_{s i} \\
& M_{\theta}=D_{s \theta} K_{s}+D_{\theta} K_{\theta}+M_{\theta i}, \\
& \varepsilon_{\theta}=\varepsilon_{s}\left(\frac{C_{s}-C_{s}}{C_{\theta}-C_{s \theta}}\right)+\frac{N_{s i}-N_{\theta i}}{C_{\theta}-C_{s}} \\
& \text { and } \\
& k_{\theta}=K_{s}\left(\frac{D_{s}-D_{s \theta}}{D_{\theta}-D_{s \theta}}\right)+\frac{M_{s i}-M_{\theta i}}{D_{\theta}-D_{s \theta}}
\end{aligned}
$$

as $\mathbf{r}$ approaches zero.

These relations are valid for both linear and nonlinear shells. 


\section{REFERIENCES}

1. K. I. Bathe, E. L. Wilson, and R. H. Iding, "NONSAP - A Structural Analysis Program for Static and Dynamic Response of Linear Systems," Dept. of Civil Eng., Univ. of CA report USCESM 74-3 (Fobruary 1974).

2. K. J. Bathe, II. Ozdcmir, and E. L. Wilson, "Static and Dynamic Geometric and Material Nonlinear Analysis," Dept. of Civil Eng., Univ. of CA report UCSESM 74-4 (Fobruary 1974).

3. P. E. Grafton and P. R. Strome, "Analysis of Axisymmetric Shells by the Direct Stiffness Method," AIAA J Vol. 1, No, 10, 2342 (October 1963).

4. R. E. Nickell and T, Sato, "Finite Element Stress Analysis of Orthotropic Layered Shells of Revolution Using a Curved Shell Element," Rohm and Haas Co., Redstone Research Lab., Technical Report S-264 (October 1970).

5. L. R. llerrmann, R. L. Taylor, and D. R. Green, "Finite Element Analysis for Solid Rocket Motor Cases," Structural Eng. Lab, Univ. of CA, Berkeley: California, Report No, 67-4 (March 1967).

6. K. L. Bathe and l:. l., Wilson, "Numerical Methods in Finite Elements Analysis," (Prentico-liall, Englewood Cliffs, New Jersey, 1976).

7. Harry kraus, Thin Elastic Shells, (John Wiley, New York, 1967).

8. S. Timoshenko and S. Woinowsky-Krieger, Theory of Plates and Shelis (McGraw-lli11, New York, 2nd Edition, 1959).

9. E. Reissner, "On the Theory of Thin Elastic Shells," in H. Relssner Anniversary Volume, (J. W. Edwards, Ann Arbor, 1949), pp. 231-247. 